\title{
Change in liana density over 30 years in a Bornean rain forest supports the escape hypothesis
}

\author{
D. M. NEWBERY (D) $1, \uparrow$ AND C. ZAHND (D) 2,3 \\ ${ }^{1}$ Institute of Plant Sciences, University of Bern, Altenbergrain 21, Bern 3013 Switzerland \\ ${ }^{2}$ Department of Life Sciences, Imperial College London, Silwood Park Campus, Ascot, Berkshire SL5 7PY UK
}

Citation: Newbery, D. M., and C. Zahnd. 2021. Change in liana density over 30 years in a Bornean rain forest supports the escape hypothesis. Ecosphere 12(8):e03537. 10.1002/ecs2.3537

\begin{abstract}
Liana dynamics may influence tree dynamics and vice versa. Only long-term studies can perhaps disentangle them. In two permanent plots of lowland dipterocarp forest at Danum, a liana census in 1988 was repeated in 2018. The primary forest was still in a late stage of recovery from an inferred large and natural disturbance in the past. Mean number of lianas per tree decreased by $22 \%$ and $34 \%$ in plots 1 and 2 , and in different ways. By 2018, there were relatively more trees with few lianas and relatively fewer trees with many lianas than in 1988. The redistribution was strongest for overstory trees of the Dipterocarpaceae (more with no lianas by 2018) and understory trees of the Euphorbiaceae (many losing high loads in especially plot 2). Proportion of trees with lianas increased overall by $3.5 \%$. The number of lianas per tree showed a quadratic relationship with tree size: maximal for large trees, and fewer for smaller and very large trees. Tree survival and stem growth rate were significantly negatively related to the number of lianas after accounting for spatial autocorrelation. Monte Carlo random subsampling of trees in 1988 and 2018, to achieve statistical independence, established significance of change. Dipterocarps and euphorbs clearly differed in their liana dynamics between plots. Regression models had different forms for the two plots, which reflected a complicated structural-spatial variability in host-liana dynamics. Analysis of the abundant tree species individually highlighted a group of emergent dipterocarps with low liana counts decreasing with time. Building on an earlier hypothesis for this forest type and site, these very large trees appear to have been losing their lianas by branch shedding, as they moved into and out of the main canopy. They were evidently escaping from the parasite. The process may in part explain the characteristically very uneven forest canopy at Danum. Change in liana density was therefore contingent on both forest history and site succession, and plot-level structure and tree dynamics. Liana promotion in the intermittent ENSO dry periods was seemingly being offset by closing of the forest and dominance by dipterocarps in late seral stages.
\end{abstract}

Key words: branch shedding; Dipterocarpaceae; forest succession; liana density and loss; story structure; tree survival and growth.

Received 22 July 2020; accepted 4 January 2021; final version received 16 March 2021. Corresponding Editor: Debra P. C. Peters.

Copyright: (C) 2021 The Authors. This is an open access article under the terms of the Creative Commons Attribution License, which permits use, distribution and reproduction in any medium, provided the original work is properly cited.

${ }^{3}$ Present address: Department of Environmental Sciences, University of Basel, Schönbeinstrasse 6, Basel, CH-4056 Switzerland.

$\dagger$ E-mail : david.newbery@ips.unibe.ch

\section{INTRODUCTION}

Lianas, or woody vines (climbers), are a conspicuous component of most tropical forests (Richards 1996). They are structural parasites:
Climbing trees allows them to reach the forest canopy with minimal investment in mechanical support. In the canopy, they compete with trees for light (Schnitzer and Bongers 2002) and thereby affect tree growth, survival, recruitment, 
and reproduction negatively (Putz 1984, Clark and Clark 1990, Phillips et al. 2005, van der Heijden and Phillips 2009, Ingwell et al. 2010). Susceptibility and tolerance to liana infestation can vary between tree species, leading to changes in forest composition, structure, and population dynamics (Hegarty 1991, Campbell and Newbery 1993, Visser et al. 2018b, Muller-Landau and Visser 2019). Lianas fit well into a broader hostparasite theoretical modeling framework (Muller-Landau and Pacala 2020).

Several studies have shown increased liana abundance in rainforests across Central and Southern America in recent decades. Phillips et al. (2002) and Wright et al. (2004) showed that abundances of lianas increased significantly in E. Amazonia and Panama, respectively. Six more studies have broadly confirmed this trend in the neotropics (Schnitzer and Bongers 2002). In the paleotropics, three studies in Africa all showed decreased liana infestation (Caballé and Martin 2001, Thomas et al. 2015, Bongers et al. 2020), as did the one only study in SE Asia (Wright et al. 2015). These four paleotropical cases though, as with the ones from the neotropics, vary so widely in their site conditions, historically contingent factors, sampling approaches, and ways of recording lianas abundance, that drawing generalizations is at present unreliable. African rain forests, for instance, like the C. and S. American ones, experience one sharp or, more often, two moderate dry seasons per year, those in SE Asia have very slight or no seasonality (Walsh 1996a).

Liana densities are most prominent in seasonal tropical forests (Gentry 1991, DeWalt et al. 2015), occur extensively in secondary successional stages of forest recovery (DeWalt et al. 2000, Wright 2005a), but also increase in large tree fall gaps in late-successional forest (Whitmore 1984, Schnitzer and Carson 2010). Three climaterelated processes or factors have been proposed to explain liana increases in neotropical primary forests, namely (1) increased rates of natural perturbation and disturbance due to droughts and storms, which open up canopies and create more gaps; (2) increased evapotranspiration due to longer, more intense, dry seasons, which favor lianas because of their deep rooting, stem anatomical adaptations, and highly efficient use of water; and (3) increased global atmospheric carbon dioxide levels, which promote the growth of lianas faster than it does of trees, especially under more lighted conditions (Schnitzer 2005, Swaine and Grace 2007, Cai et al. 2009, Schnitzer and Bongers 2011).

Lianas are nevertheless an important feature of aseasonal and less-recently disturbed rain forests in the paleotropics, particularly in SE Asia where primary forests are composed in recent decades largely of late-successional stages (Ashton 1964, 2014, Ashton and Hall 1992, Hazebroek et al. 2012). Liana abundance results here from the outcome of recent decades of structural stability combined with, in places, the legacy of major destabilizing natural events in the past (Newbery et al. 1992, 1999). When such a forest is in the late stages of recovery after a historic major disturbance, a decline in liana abundance is expected (Campbell and Newbery 1993). The reasoning behind this postulate is that, as tree basal area abundance tends toward a site maximum, the main canopy closes, gaps become fewer, and light conditions internal to the stand will be less favorable for liana recruitment and growth (Whitmore 1984).

With dry season seasonality and associated strong drought not being the issue, two factors remain though that could counteract an endsuccession decline in SE Asian forests, namely a continuing rise in atmospheric carbon dioxide (Malhi and Phillips 2005), and perturbations caused by the intermittent effects of the El Nino Southern Oscillation (ENSO; Walsh 1996b, Wright 2005b) which open the canopy and lead the understory to temporarily have more lighted and drier conditions (Walsh and Newbery 1999, Newbery et al. 2011). Lianas are also extensively distributed in logged forests of SE Asia (e.g., Pinard and Putz 1994), and have a major effect on forest regeneration, but that is a separate process from the dynamics of the mature primary forests, except for when these forests were last heavily disturbed by similar-scale natural events, for example, by extensive drought and/or fire (Beaman et al. 1985).

Wright et al. (2015) reported on changes in liana abundance at Pasoh, Peninsular Malaysia, between 2002 and 2014, in terms of canopy cover infestation on trees $\geq 30 \mathrm{~cm}$ dbh $(95.4 \mathrm{~cm}$ gbh). They confirmed the lower susceptibility of dipterocarps to lianas compared with other tree families shown by Campbell and Newbery (1993). 
Tree mortality for all trees was affected when liana cover exceeded $75 \%$. The change in proportions of all survivor trees infested was very small, declining from $52.3 \%$ to $47.9 \%$. The frequency of trees with $\geq 50 \%$ cover of lianas barely altered. The samples were not independent though, because of the surviving trees in common.

An analysis of the change in liana infestation over a 30-yr period in a late-successional primary lowland dipterocarp forest in NE Borneo is presented here to test expectations that liana infestation decreased over time, but lianas were still having negative effects on tree growth and survival. The hypothesis of Campbell and Newbery (1993), that liana loss is largely controlled by branch fall for overstory trees and whole tree death for understory ones, is extended to show that this mechanism allows the dipterocarps, especially, to escape their parasitic liana load when reaching the upper canopy and becoming emergent.

\section{Methods}

\section{Study site}

This study was conducted in two permanent plots of primary lowland dipterocarp rainforest within the Danum Valley Conservation Area (DVCA) in Sabah, northeastern Borneo, Malaysia (Marsh and Greer 1992). The plots are each 4 ha $(100 \times 400 \mathrm{~m})$, lying parallel $\sim 280 \mathrm{~m}$ apart and about $800 \mathrm{~m}$ west of the Field Centre $\left(4^{\circ} 57^{\prime} 48^{\prime \prime} \mathrm{N}, 117^{\circ} 48^{\prime} 10^{\prime \prime}\right.$ E; Newbery et al. 1992). The area is $\sim 230 \mathrm{~m}$ above sea level, and both plots feature a $\sim 35 \mathrm{~m}$ change across gently undulating terrain. The climate is mostly aseasonal, with an average monthly temperature of $26.9^{\circ} \mathrm{C}$ and an average annual rainfall of $2832 \mathrm{~mm}$ (1986-2007, Newbery et al. 2011). More detailed information is given in Newbery et al. (1992, 1996) and Walsh and Newbery (1999). The two plots are designated main plots, MP1 and MP2, at Danum to distinguish them from 10 smaller neighboring satellite ones.

The permanent plots were set up between 1985 and 1986 and have since been under ongoing long-term observation (Newbery et al. 1992). All trees with a girth at breast height $\geq 10 \mathrm{~cm}$ (gbh, at $1.3 \mathrm{~m}$ or above buttresses) were tagged, mapped, and identified, and gbh was measured. Since then, four more censuses have been made, the latest in 2015 (D. M. Newbery, P. Stoll, and M. Lingenfelder, unpublished data). In each of those censuses, survivors, recruits, and dead trees were recorded and gbh was measured for all live trees.

The two plots had been initially intended as replicate areas of the forest locally, especially in terms of their species richness, general structure, and biomass (Newbery et al. 1992). However, over time species' tree size distributions diverged and the plots differed in their dynamics (Newbery et al. 1999, Lingenfelder and Newbery 2009; D. M. Newbery, P. Stoll, and M. Lingenfelder, unpublished data). Thus, for the present liana study spanning $30 \mathrm{yr}$, unless otherwise indicated, the plots were treated separately. Subsequently, this proved valuable as it led to much better insights into the liana dynamics than would have been achieved by a combined analysis, one that used them as simply statistical replicates.

\section{Fieldwork}

From September 1987 to February 1988 and June to September 1989 (mid-date September 1988), a first liana census in the two plots was conducted by Campbell and Newbery (1993). Liana stems on all trees $\geq 30 \mathrm{~cm}$ gbh were counted. The present study in June and July 2018, 30 yr later, closely followed the procedure of the first one to achieve the highest feasible level of compatibility (Zahnd 2018). Liana stems $\geq 2 \mathrm{~cm}$ gbh on all trees $\geq 30 \mathrm{~cm}$ gbh were again counted. Lianas were defined here as woody climbing plants, which start growing from the ground. Lianas were regarded as individuals when they rooted close to the tree observed, or when crossing over into the target tree from another tree. Only lianas that had foliage in the canopy of the tree were counted: those that did not reach the crown of the tree, or only made a loop and descended, or that were clearly dead, were not counted. As in the 1988 census, lianas were not identified taxonomically because taking botanical samples for identification would have influenced the growth and mortality of the trees themselves undergoing long-term observation (Campbell and Newbery 1993).

For the present study, tree girths measured in the first (1986) and last (2015) main plot tree censuses were applied to the liana counts from 1988 and 2018. These censuses gave the tree data closest in terms of plot census dates to those of the 
liana censuses. For both liana censuses, trees that died between 1986 and 1988 or between 2015 and 2018, respectively, were disregarded, as were trees that only grew bigger than $30 \mathrm{~cm}$ gbh in the respective periods. By happenstance, the liana censuses were both at intervals of 2-3 yr after the tree censuses, so if the dynamics in these relatively short periods can be taken as being equivalent there should have been very little bias introduced from the census matching. In this paper, data collected in 1986 and 1988 will be collectively referred to as "census 1" (or "date 1" in the analysis), and those collected in 2015 and 2018 as "census 2" (or "date 2").

The species codes in the 2017-digitized appendices of Campbell (1990), which formed the basis to the second liana census, were made fully compatible with the taxonomy of the last (fifth) 2015 plot census. Since the first census in 1986 (Newbery et al. 1992), some tree identifications of survivors were corrected (the more important ones mentioned in Newbery et al. 1999), with the consequence that for a few of them family membership changed, and liana counts per tree in the different families reported in this paper may differ very slightly from those given by Campbell and Newbery (1993). Other tree names changed as a result of taxonomic revisions. Twenty-eight trees in four taxa changed names, but all remained in the same families. Within-plot elevations (ground height above plot origin) were available for all trees $\geq 30 \mathrm{~cm}$ gbh in 1986, and the 35 and 46 trees in MP1 and MP2, respectively, recruiting into this size class by 2015 were found by interpolation from the plot coordinates of nearest-neighbor survivors (Lingenfelder and Newbery 2009).

\section{DATA ANALYSIS}

In analyzing the changes in liana densities per tree between censuses 1 and 2, plots MP1 and MP2 were handled separately. Since the plots differed in topographic variation, tree size structure, forest dynamics, and species composition, even in moderately small ways, combining them into one sample, would have confounded several important and interesting differences that bear on the relationships between liana density and dynamics and these factors. Moreover, the plots are replicates at the 4-ha scale and separated enough spatially to provide some indication of forest variability on the local landscape. Apart from revisions to nomenclature, the 1988 liana data used here are the same as those reported in Campbell and Newbery (1993).

Trees at each census were divided into four size classes: (1) 30 to $<60$; (2) 60 to <120; (3) 120 to $<240$; and (4) $\geq 240 \mathrm{~cm}$ gbh. These are referred to as medium-sized, large, very large, and largest trees, respectively: The class small would be those 10 to $<30 \mathrm{~cm}$ gbh, not considered for the liana censuses. Over-understory index (OUI, scale $0-100)$, for the 100 most abundant species in the plots, was adopted from Newbery et al. (2011). It is based on first axis of principal component analyses of ratios of numbers of trees $\geq 30 \mathrm{~cm} \mathrm{gbh} / \geq 10 \mathrm{~cm}$ gbh and ratios of basal areas of the same size classes, the rescaled scores averaged over four plot-tree censuses 1986-2007. Three stories were designated: overstory (OUI > 55), intermediate (OUI 20 to <55), and understory (OUI <20). The $30 \mathrm{~cm}$ gbh threshold is the same as the lower one for liana recording.

Analyses of the liana census data were mainly done in the computing language $\mathrm{R}$ (Fox and Weisberg 2011, R Core Team 2020). Special commands are listed in Appendix S5, where part 1 has the basic code for randomization testing of differences over time, and part 2 lays out the essential regression models, particularly those that allowed for spatial autocorrelation.

\section{Error distributions}

The negative binomial distribution fitted the frequencies of number of lianas per tree far better than the Poisson, with $\chi^{2}$ values of 19.34 and 14.41 in 1988 for MP1 and MP2, respectively $(\mathrm{df}=7)$, and correspondingly 13.89 and 14.50 in $2018(\mathrm{df}=5)$. All four cases were nevertheless significant with $P$ between 0.01 and 0.05 . For comparison, when fitting the Poisson, $\chi^{2}$ values were 3344 and 1185 in 1988 and 2018 (plots together). The $k$ values (expressing degree of aggregation when $<1$ ) were, for the four cases in order, 0.625, $0.491,1.265$, and 1.034 , indicating that lianas were much more aggregated in 1988 than in 2018. The geometric Poisson (Polya-Aeppli) distribution gave very similar fits to the negative binomial, and since the latter is fairly widely incorporated as a family error distribution in most regression software, it was taken to be the error model. The 
remaining differences in fit were largely due to observed frequencies being less than expected ones for counts of 1 and 2 and the converse for counts of 3 and 4, for which no single-mode function can readily cater.

\section{Numbers of lianas}

Negative binomial GLM regressions were made using the glm.nb function in MASS library of R. Comparing fits (by anova) of nlianas $\sim \ln$ (gbh) and nlianas $\sim \ln (\mathrm{gbh})+$ elev for 1988 led to LRs (log-likelihood ratios) of $11.86(P \leq 0.001)$ and $9.32(P \leq 0.01)$ in MP1 and MP2, respectively, and correspondingly for 2018 LRs of 13.34 and 40.43 (both $P \leq 0.001$ ), indicating that including elevation enhanced the model significantly. Here and later in model formulations, the term nlianas is number of lianas (or no. lianas) per tree. Adding a $\ln (\mathrm{gbh}) \cdot \mathrm{elev}$ interaction to the two-term model led to no improvement, LRs of 0.14 and 0.49 in 1988 and 0.02 and $<0.01$ in 2018 (all $P>0.10$ ). By contrast, including instead a $[\ln (\mathrm{gbh})]^{2}$ term led in 1988 to LRs of $3.10(P \leq 0.1)$ and $31.87(P \leq 0.001)$ in MP1 and MP2, and 2018 LRs of 31.34 and 56.64 (both $P \leq 0.001$ ). The final model was therefore nlianas $\sim \ln (g b h)+[\ln (g b h)]^{2}+$ elev. Fitting the best model resulted in dispersion values of 0.948 and 1.001 in MP1 and MP2 for 1988, and 1.011 and 0.938 correspondingly for 2018. Diagnostic plots of Pearson's residuals vs. fitted showed reasonable spreads of points, with the typical striations for discrete data: Residuals themselves, however, were positively skewed.

To test and cater for spatial autocorrelation, hlikelihood GLM (HGLM) was run using the hglm function in same-named hglm library of $\mathrm{R}$, by including a SAR correlation term based on plot inverse distance matrices. As hglm does not cater for a negative binomial error, this had to be substituted by a quasi-Poisson one, and models without the spatial autocorrelation adjustments compared with the negative binomial one. Running the selected model with the interaction also resulted in later being insignificant for all census $x$ plot combinations. The method is described in Alam et al. (2015), Lee et al. $(2017 a, b)$, and Ronnegard et al. (2010).

\section{Tree survival}

Tree survival between 1988 and 2018 was modeled with a logistic GLM regression (Hilbe 2009,
2011), as a standard glm (binomial error) starting with survival $\sim \operatorname{sqrt}\left(\right.$ nlianas $\left._{88}\right)$. The transformation normalized the original nlianas variable to a large extent but not entirely. Adding $\ln \left(\mathrm{gbh}_{88}\right)$ and elevation resulted in varying degrees of improvement to the fits depending on the plot. With $\ln (\mathrm{gbh})$, the LRs were $4.50(P \leq 0.05)$ and $2.67(P>0.10)$ in MP1 and MP2, respectively, while adding elevation led correspondingly to LRs of $0.02(P>0.05)$ and $7.47 \quad(P \leq 0.01)$, although including both terms they were 4.58 $(P \leq 0.05)$ and $10.37(P \leq 0.01)$. As a compromise, in order to compare plots on a same-model basis, all three terms were retained to have a final model: survival $\sim \operatorname{sqrt}\left(\right.$ nlianas $\left._{88}\right)+\ln \left(\mathrm{gbh}_{88}\right)+$ elev.

To account for spatial autocorrelation, an autologistic regression was run with the command logistic.regression in the library spatialEco in $R$, using an inverse squared-distance matrix as a measure of correlation. Further, as an alternative to handling spatial autocorrelation affecting the model, repeated stratified sampling was achieved by taking one tree at random per $20 \times 20 \mathrm{~m}$ subplot (100 trees per plot), $N^{\prime}=500$ times, and averaging the coefficients and statistics. This meant that trees on average $20 \mathrm{~m}$ apart were assumed to have independent liana counts. The repeated runs will have reused trees many times and not be independent, especially when there were very few per subplot from which to sample. This additional approach was intended to be confirmatory of the regressions, free of a distance-decay function. Methods are outlined and discussed in Besag (1972), Cliff and Ord (1981), Augustin et al. (1996), Dormann (2007), Zuur et al. (2009), and Evans (2018).

Analysis at the family level, for the Dipterocarpaceae and Euphorbiaceae, failed to converge to a solution with the autologistic function in three of four cases, and it was replaced by a GLMM using the coordinates of $20 \times 20 \mathrm{~m}$ subplots as the cluster term in command glmmML of the library glmmML in R (Rhodes et al. 2009, Brostrom and Holmberg 2011).

\section{Tree growth}

With standard linear, or equivalently generalized least squares (GLS), regression (Gaussian error) as the function $\mathrm{lm}$ in $\mathrm{R}$, relative growth rates $\left(\mathrm{rgr}, \mathrm{mm} \cdot \mathrm{m}^{-1} \cdot \mathrm{yr}^{-1}\right.$ ) of trees, that is survivors, between 1988 and 2018, were fitted first 
as $\sim \operatorname{sqrt}\left(\right.$ nlianas $\left._{88}\right)$. Adding the term $\ln \left(\mathrm{gbh}_{88}\right)$ considerably improved model fits, with change in $F$ ratio (equivalent to LR) of 39.81 and 30.51 (both $P \leq 0.001$ ) in MP1 and MP2, respectively, but not consistently so much in the case of elevation, the corresponding LRs being $3.60(P \leq 0.1)$ and $0.62(P>0.10)$ : Both terms included led to combined improvements over sqrt(nlianas 88 ) alone, LRs of 21.08 and 15.53 (both $P \leq 0.001$ ). Adding the squared size term, $\left[\ln \left(\mathrm{gbh}_{88}\right)\right]^{2}$, to $\ln$ $\left(\mathrm{gbh}_{88}\right)$ and sqrt(nlianas $\left.{ }_{88}\right)$ was of little gain, with $F$ ratios of 0.10 and 2.10, and adding to these elevation, 1.20 and 1.28 (all four at $P>0.10$ ). Attempting to cater for some remaining nonnormality in rgr, by using gamma or inverse Gaussian errors, resulted in scarce improvement in either fits or residual plots. Accordingly, the best parsimonious model was $\mathrm{rgr} \sim$ sqrt $\left(\right.$ nlianas $\left._{88}\right)+\ln \left(\right.$ gbh $\left._{88}\right)$.

With a rgr regression using the Gaussian error, there were more ways of dealing with spatial autocorrelation than those (currently as software) available for regressions with binomial and negative binomial errors. Firstly, spatial dependence was modeled using a variogram of exponential form in GLS (a correlation structure), within the function gls in library nlme in R. Comparing the accepted model form, without and with the spatial correlation term, led to modest improvement in the fits, with LRs of $8.84(P \leq 0.05)$ and 5.54 $(P \leq 0.10)$ for MP1 and MP2, respectively. Using a spherical form in the variogram made hardly any difference. Randomization runs were conducted in the same way as they were for the survival data. A different, additional approach was to use a correlation matrix again based on inverse distances but guided by prior analysis of spatial autocorrelation via a correlogram. Two indices, Moran's I and Geary's C, were calculated at 5-m interval distances between 0 and $40 \mathrm{~m}$. Moran's I for MP1 was significant at 5-10 m $(P \leq 0.001)$ and at $30-35 \mathrm{~m}(P \leq 0.1)$, and for MP2 at $0-5 \mathrm{~m}(P \leq 0.1), 10-15$, and $15-20 \mathrm{~m}$ (both $P \leq 0.05)$. Geary's $C$ was significant in MP1 at $0-5 \mathrm{~m}(P \leq 0.01)$ and $5-10 \mathrm{~m}$ too $(P \leq 0.05)$, and in MP2 again at $0-5 \mathrm{~m}(P \leq 0.001)$ and $15-$ $20 \mathrm{~m}(P \leq 0.10)$. Thus, up to $20 \mathrm{~m}$, both indices show significant spatial correlation, permitting use of the spautolm function in library spatialreg in $\mathrm{R}$, with SAR mode and range $0-20 \mathrm{~m}$. The methods used here follow Pinheiro and Bates
(2000), Haining (2010), Plant (2012), Bivand et al. (2013), and Borcard et al. (2018).

\section{Change in liana abundance with time}

Changes in the proportion of trees carrying lianas, the frequencies of trees in liana density classes, and the mean number of lianas per tree, between 1988 and 2018, were estimated. Some of the trees were different ones at the two dates (those which died after 1988 were lost and those which recruited after 1988 to survive to 2018 were gained), but close to half were the same individuals (the survivors). (The 1721 survivors formed $51.2 \%$ of the trees in 1988 and $48.7 \%$ of those in 2018.) Therefore, the tree populations at the two dates were not fully statistically independent. Subsamples of different trees were accordingly selected for 1988 and for 2018, by taking at random half of those in 1988 (a uniform distribution of the tag numbers), flagging them, and the unflagged trees contributed to the sample for 2018. Some unflagged trees will not have survived 1988 to 2018, and they were replaced (approximately in terms of numbers) by recruits appearing in 2018. Thus, no one tree was present in both dates' subsamples. Statistical tests were repeated $N^{\prime}=500$ times as a Monte Carlo procedure (Manly 1997). This randomization procedure not only eliminated temporal dependence but also reduced the effects of spatial autocorrelation.

Differences in the proportions of trees having lianas, that is presence or absence of lianas, at the two dates were tested using Pearson's $\chi^{2}$ statistic $(2 \times 2$ test; $\mathrm{df}=1)$. For frequencies of trees in liana density classes $(0,1,2 \ldots r$ lianas $)$, the $\chi^{2}$ statistic was again applied $(2 \times r$ test, $\mathrm{df}=r-1$ ), with the provision, achieved by pooling higher tail classes, that no class had $<5$ trees. Firstly, all trees were used in single tests, without any subsampling. Randomization tests were then run $N^{\prime}=500$ times, the mean statistic found and its significance, and the null hypothesis was rejected overall when $\geq 80 \%$ of the randomization runs were individually significant at the $P \leq 0.05$. This procedure admits a power of 0.8 and type II error rate of $\beta=0.2$ (Cohen 1988, 1992). The mean $\chi^{2}$ values were taken as representing typical differences in proportions free of any temporal autocorrelation effects. Additionally, McNemar's $\chi^{2}$ test of symmetry (Agresti 2007) was employed to ask whether 
proportions of trees with or without lianas changed with date, for just the 1988 survivors. The symmetry statistic could only be calculated for counts in the density classes when taking all trees: The two main families had insufficient numbers.

Change in proportions of trees with lianas between the two dates could be analyzed too with the negative binomial GLM, to ask whether mean numbers per tree differed significantly, and whether the relationship between proportion and size of tree changed over time. Regressions were run with subsamples at the two dates from the same $N^{\prime}=500$ randomizations; they compared nested models 1 to 4: nlianas $\sim$ year, year $+\ln (\mathrm{gbh}), \sim$ year ${ }^{*} \ln (\mathrm{gbh})$, and $\sim$ year ${ }^{*} \ln$ $(\mathrm{gbh})+[\ln (\mathrm{gbh})]^{2}+$ year: $[\ln (\mathrm{gbh})]^{2}$. The last two models tested whether differences in proportions were further accountable for by linear and nonlinear interactions between year and $\ln (\mathrm{gbh})$. Interpreting the interactions in GLMs between a categorical factor (e.g., year) and continuous variables $\left(\ln [\mathrm{gbh}]\right.$ and $\left.[\ln (\mathrm{gbh})]^{2}\right)$, and then using the term to infer estimates of the expected response variable (nlianas), requires special consideration. Guidance to the calculations is found in Hilbe (2009), Cameron and Trivedi (2013), and Long and Freese (2014).

\section{Liana abundance and dynamic status}

Starting with the trees censused in 1988, proportions of trees with lianas could be compared between those dying and those surviving by 2018 with the $\chi^{2}$ test of association, and the numbers of lianas per tree similarly compared with negative binomial GLM. In a complementary way, those trees in 2018 that were survivors from 1988 and those that had recruited by then (into the $\geq 30 \mathrm{~cm}$ gbh population) could also be compared.

\section{Liana densities per tree for individual species}

Mean number of lianas per tree was found for the 51 and 52 species in 1988 and 2018, respectively, with $\geq 20$ trees per species, from fitting individual negative binomial distributions. Overunderstory index (OUI) values were already available for the 100 most abundant species. Six further species occurred in a list of 100 for 2015, and their OUI values were taken as the means of the genera to which they belonged. Mean gbh per species was derived from the main plot census records. Stem rgr in girth of small trees (10 to $<50 \mathrm{~cm}$ gbh) for period 1, between the first two tree censuses (1986-1996), have been reported in Newbery et al. (1999), though the 100 most abundant species (N/plot $\geq 10 \mathrm{~cm}$ gbh) available for here are the very slightly revised values of 2010, and were for only valid, that is, unproblematic, gbh estimates (Lingenfelder and Newbery 2009). In this way, 45 and 47 (of the 51 and 52) species could be matched with rgr values averaged across the two plots.

\section{Results}

\section{Background tree dynamics}

At census 1 (1986|1988), there were 1704 and 1659 trees of $\geq 30 \mathrm{~cm}$ gbh in MP1 and MP2, respectively (together 3363), and by census 2 (2015|2018) correspondingly 1699 and 1832 (3531), discounting the few that died between matched tree and liana censuses. Between the censuses, 926 and 716 (together 1642) trees died in MP1 and MP2, and correspondingly 921 and 889 (1810) trees recruited into the size class. The full data set consisted then of $3363+1810=5373$ trees involved in both censuses. The annualized mortality rates were accordingly $2.58 \%$ and $1.87 \%$, and the annualized recruitment rates were $1.45 \%$ and $1.33 \%$, in MP1 and MP2, respectively. (Mortality was based on the proportions of the numbers of trees alive in 1986 dying by 2015, while recruitment was based on proportions of trees alive in 2015, which had joined the population since 1986; see Newbery and Lingenfelder 2009 for Danum). Thus, mortality rate was higher than recruitment in both plots, and both mortality and recruitment rates in MP1 were higher than their corresponding values in MP2, especially for mortality (38\% higher). In the $30 \mathrm{yr}, \mathrm{MP} 1$ had a $26 \%$ higher turnover than did MP2 (2.02 vs. 1.60\%). Overall, trees $\geq 30 \mathrm{~cm}$ gbh occurring in both censuses and both plots belonged to 355 species, 143 genera, and 55 families. The frequency distributions of tree gbh were very similar between plots and dates, especially close for MP1 (Appendix S1: Fig. S1).

\section{Liana frequency distributions}

The nine to ten most abundant tree families (Table 1 and Appendix S1: Table S1) combined 
Table 1. Number of trees, relative abundance, proportion of liana-infested trees, and average number of lianas per tree for all trees and those in the two most abundant families, at each census in main plots MP1 and MP2.

\begin{tabular}{|c|c|c|c|c|c|c|}
\hline \multirow[b]{2}{*}{ Tree family } & \multicolumn{2}{|c|}{$\begin{array}{c}\text { No. } \\
\text { trees/plot }\end{array}$} & \multicolumn{2}{|c|}{$\begin{array}{l}\text { Trees with } \\
\text { lianas (\%) }\end{array}$} & \multicolumn{2}{|c|}{$\begin{array}{l}\text { Average no. } \\
\text { lianas/tree }\end{array}$} \\
\hline & 1988 & 2018 & 1988 & 2018 & 1988 & 2018 \\
\hline \multicolumn{7}{|l|}{ MP1 } \\
\hline All trees & 1704 & 1699 & 60.3 & 65.0 & 2.164 & 1.686 \\
\hline Dipterocarps & 246 & 247 & 45.9 & 40.1 & 1.841 & 1.000 \\
\hline Euphorbs & 206 & 190 & 64.6 & 68.9 & 1.922 & 1.889 \\
\hline \multicolumn{7}{|l|}{ MP2 } \\
\hline All trees & 1659 & 1832 & 54.3 & 56.7 & 2.017 & 1.333 \\
\hline Dipterocarps & 330 & 353 & 36.7 & 36.8 & 1.361 & 0.841 \\
\hline Euphorbs & 219 & 225 & 53.0 & 53.3 & 1.995 & 1.053 \\
\hline
\end{tabular}

made up $75 \%$ and $71 \%$ of all trees in MP1 and MP2, respectively. The most abundant families were the Dipterocarpaceae and the Euphorbiaceae, followed by the Meliaceae, Lauraceae, Phyllanthaceae, and Annonaceae. Relative abundances of the first 13 families per plot plus all others pooled into a 14th did not change significantly between dates (MP1 $\chi^{2}=9.57, \mathrm{df}=13$, $P=0.73$; MP2 $\left.\chi^{2}=18.05, \mathrm{df}=13, P=0.16\right)$, nor did the overall $10-\mathrm{cm}$ gbh classed distributions of all trees (pooling trees $\geq 150 \mathrm{~cm}$ ) between years (MP1 $\chi^{2}=14.19, \quad \mathrm{df}=12, \quad P=0.286 ; \quad$ MP2 $\chi^{2}=9.35, \quad \mathrm{df}=12, \quad P=0.67 ; \quad$ see $\quad$ also Appendix S1: Fig. S1). This largely rules out that changes in overall liana density were due to major shifts in the tree community over the 30 yr. Nevertheless, MP1 and MP2 did differ in one important respect: MP1 had more very large trees than MP2 (Appendix S1: Fig. S1), although they decreased in number more in MP2 than in MP1 between 1988 and 2018. These very large trees were mostly dipterocarps.

In total, 2865 and 2442 liana stems were counted in plots 1 and 2, respectively. These are $22.3 \%$ and $27.0 \%$ decreases since the first census (3688 and 3347 lianas in plots 1 and 2, respectively). The mean numbers of lianas per tree, excluding trees without any lianas, decreased in MP1 and MP2 from, respectively, 2.16 and 2.02 in the first census to 1.69 and 1.33 in the second one, decreases of $21.8 \%$ and $34.2 \%$ (Table 1). For all trees, the proportion of liana-infested trees significantly increased by $4.7 \%$ in MP1 and by $2.4 \%$ in
MP2. None of the major families individually showed significant changes in the proportions, although in MP1 trees in the Dipterocarpaceae decreased, while those in the Euphorbiaceae correspondingly increased: Differences for these two families in MP2 were negligible (Table 1).

Frequency distributions of counts of lianas per tree declined smoothly in plots MP1 and MP2, having relatively more trees with no or few (1-3) lianas, and relatively fewer trees with many $(\geq 7)$ lianas in 2018 than in 1988 (Fig. 1a, b). The distributions appeared approximately exponential in form. For the Dipterocarpaceae, inflation of zero counts was obvious, with the difference between years matching the overall one in MP2 but not so well in MP1 (Fig. 1c, d). The Euphorbiaceae followed a scaled-down representation of the overall plot distributions in MP2 (also for the zero class), but MP1 not (Fig. 1e, f). The two plots appeared on first examination to be showing different dynamics in liana densities over time, which justified them being separately analyzed statistically. The liana distributions for 1988 are the same as those used in Campbell and Newbery (1993: Figs. 2, 3), albeit presented here slightly differently. In that earlier paper, the size distributions of trees in the Dipterocarpaceae and Euphorbiaceae were compared (Campbell and Newbery 1993: Fig. 1).

Considering the frequency distributions of numbers of lianas per tree in the four size classes (Fig. 2), the gradual decline for 0 to $\geq 8$ lianas per tree, in both plots and at both dates, in the medium-sized trees (class 1) becomes increasingly more discontinuous as proportionally more trees up to the very large trees (class 3 ) have no lianas, and among the largest (class 4) zero counts become dominant. This implies, indirectly, that as the trees become larger they are shedding their lianas.

\section{Number of lianas and tree size}

The relationship between number of lianas per tree and tree size (gbh) could be described by a quadratic form, for MP1 in 1988 rather weakly, but more strongly in 2018 (Table 2, Fig. 3). For MP2, the fits were similarly strongly quadratic at both census dates. More lianas were recorded on medium than on small and large trees: The distinction between censuses in MP1 appeared mainly due to large trees in 1988 having similar 
(a) All - MP1

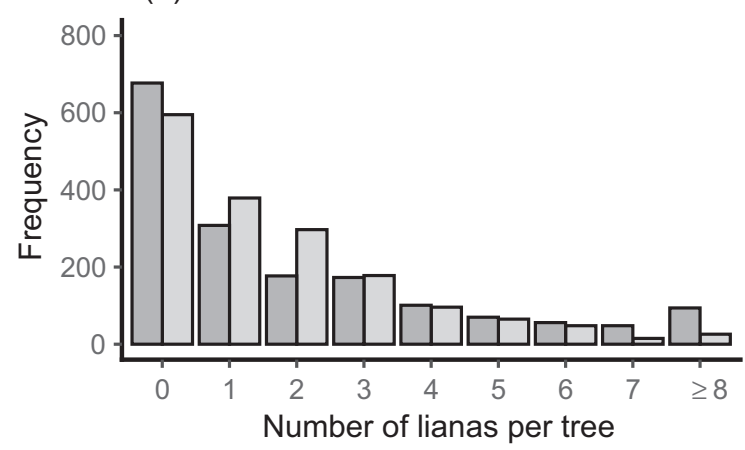

(c) Dipt - MP1

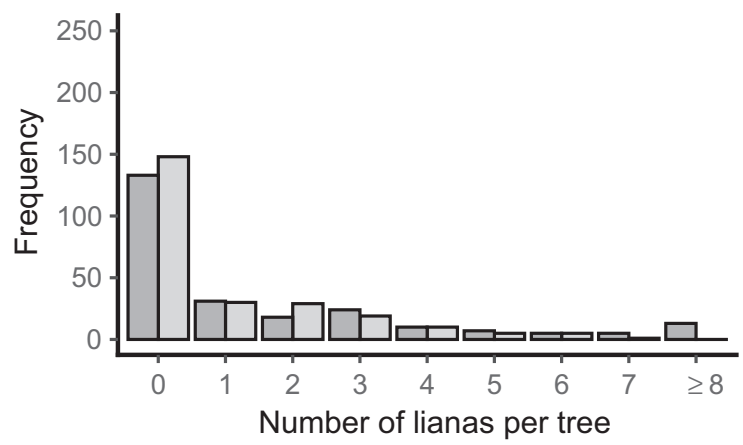

(e) Euph - MP1

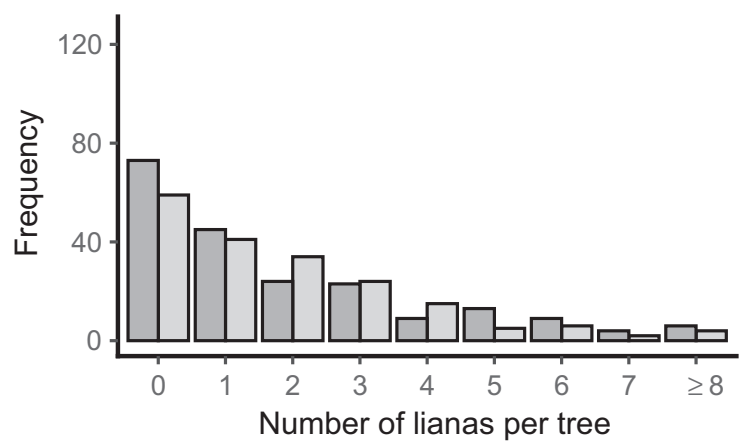

(b) All - MP2

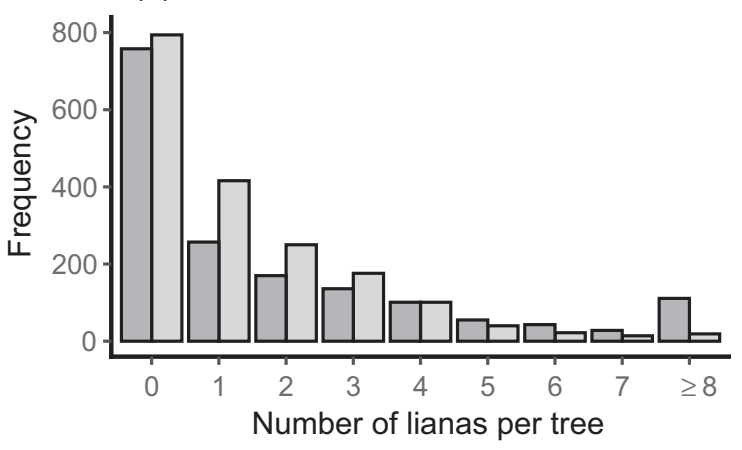

(d) Dipt - MP2

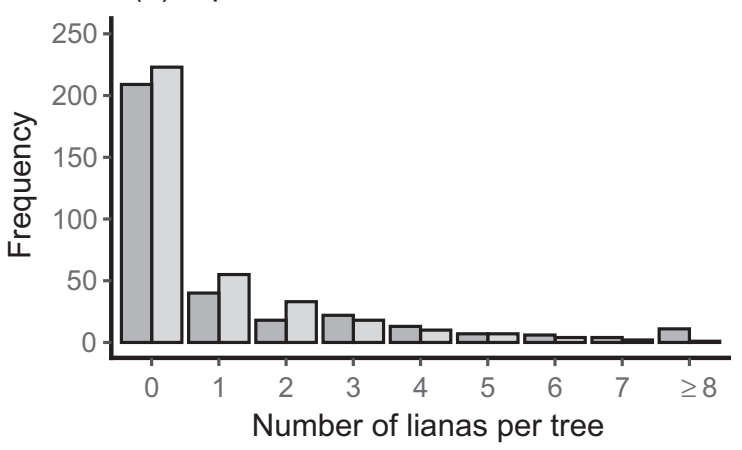

(f) Euph - MP2

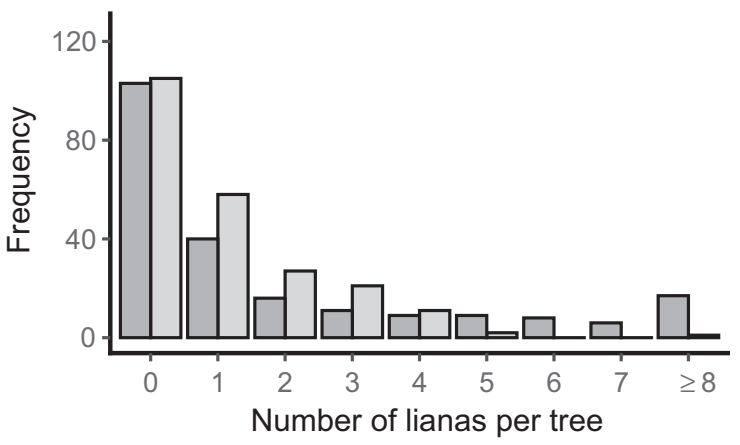

Fig. 1. Frequency distributions of trees in classes of numbers of lianas per tree in 1988 (dark gray bars) and in 2018 (light gray bars) in the two main plots MP1 and MP2 at Danum, for trees in (a, b) all families, (c, d) Dipterocarpaceae, and (e, f) Euphorbiaceae.

loads to medium-sized ones. Consequently, change in numbers over time was more marked for MP1 than for MP2. In 1988, coefficients were statistically weaker for MP1 than for MP2; in 2018, they were highly significant for both plots. Adjustment for spatial autocorrelation was small although its effect was more noticeable for MP1 in 1988, especially on the gbh terms. Regression using HGLM with the quasi-Poisson error gave similar results to the negative binomial GLM (Appendix S2: Tables S1a, b), suggesting that the variance-mean relationship in numbers of lianas was close to linear (see ver Hoef and Boveng 2007).

Pseudo- $R^{2}$ values (as \%), based on differences in deviances for fitted and null models (in the 
(a) Size class 1 - MP1

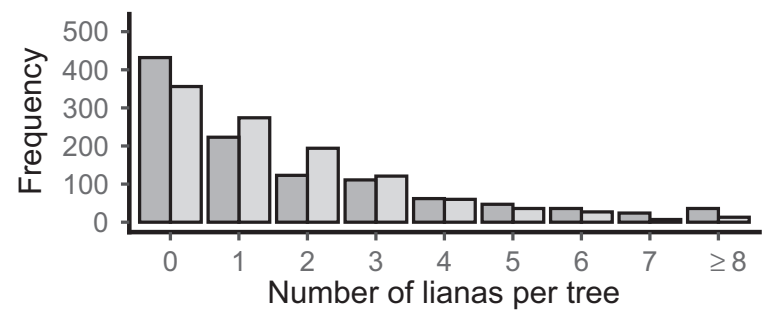

(c) Size class 2 - MP1

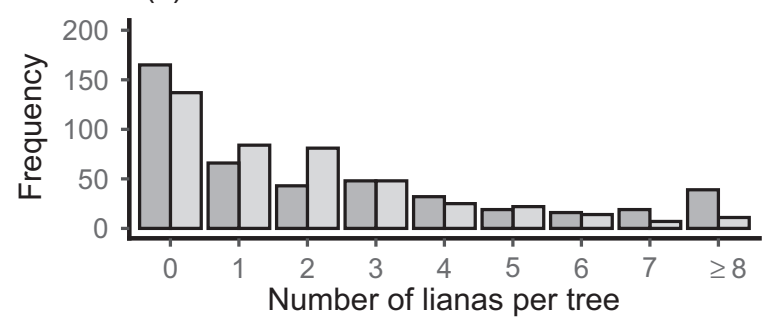

(e) Size class 3 - MP1

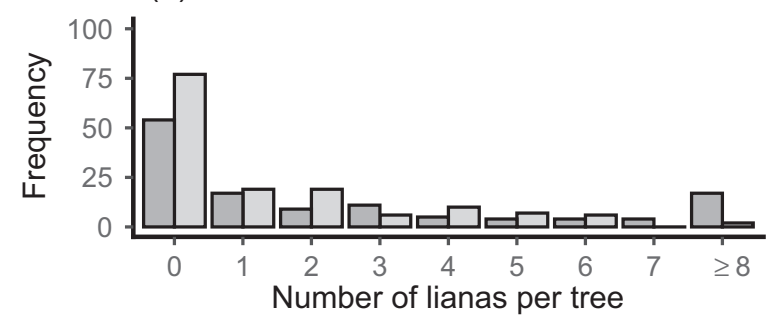

(g) Size class 4 - MP1

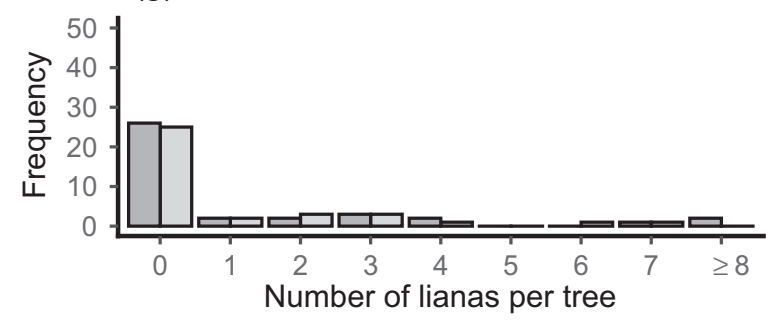

(b) Size class 1 - MP2

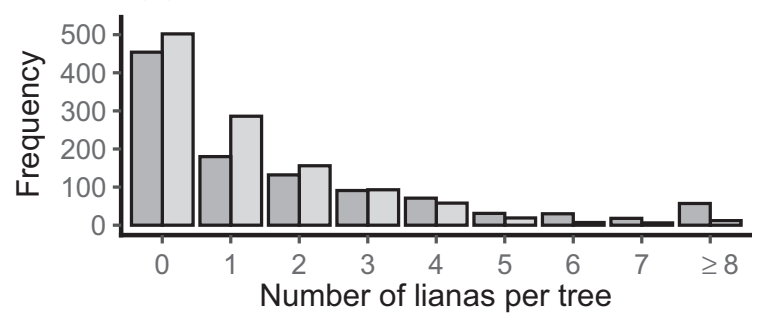

(d) Size class 2 - MP2

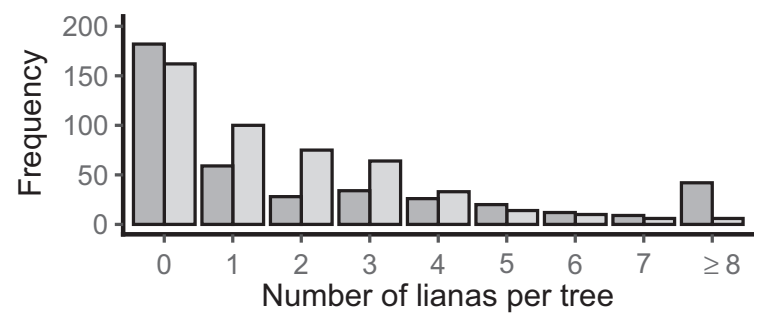

(f) Size class $3-$ MP2

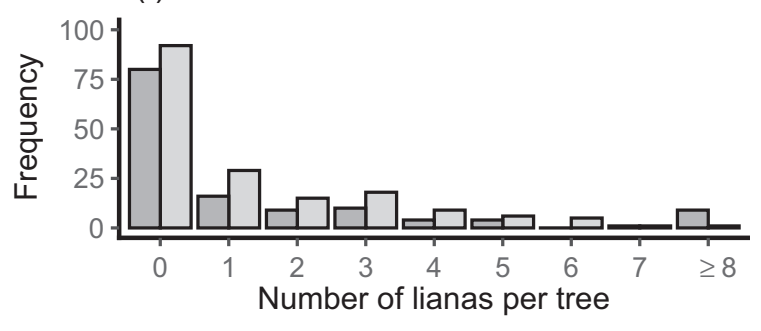

(h) Size class $4-$ MP2

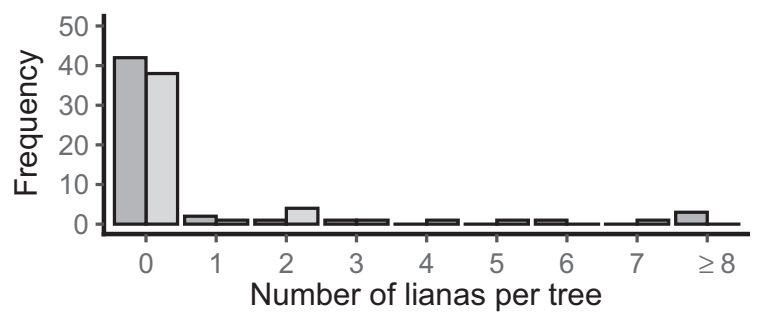

Fig. 2. Frequency distributions of trees in classes of numbers of lianas per tree in 1988 (dark gray bars) and in 2018 (light gray bars) in the two main plots MP1 and MP2 at Danum, for trees in four tree girth size classes (gbh): (a, b) 1, 30 to $<60 \mathrm{~cm}$; (c, d) 2, 60 to $<120 \mathrm{~cm}$; (e, f) 3, 120 to $<240 \mathrm{~cm}$; and 4, $\geq 240 \mathrm{~cm}$.

latter the mean was the sole term), and their random and fixed components (in parenthesis) were, in 1988, $24.2(22.0,2.2)$, and $16.8(14.8,2.0)$ for MP1 and MP2, respectively, and correspondingly in 2018, 20 (17.4 and 2.6) and $10.2(4.6,5.6)$. The statistically weaker terms for MP1 in 1988 match with the highest, whereas the strongest terms in MP2 in 2018 had the lowest, random component. Even so, the fixed component was small at just $2-6 \%$. The model fits are descriptive, and not fully hypothesis testing, because $\ln (\mathrm{gbh})$ and $[\ln (\mathrm{gbh})]^{2}$ were not statistically independent variables (i.e., some collinearity was present). It follows that the absolute values of the $t$-statistic 
(a) 1988 MP1

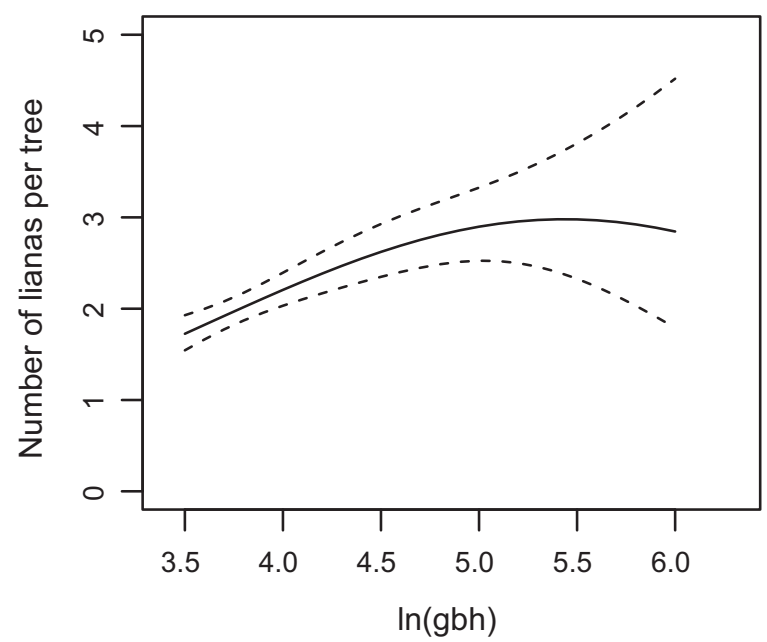

(c) 1988 MP2

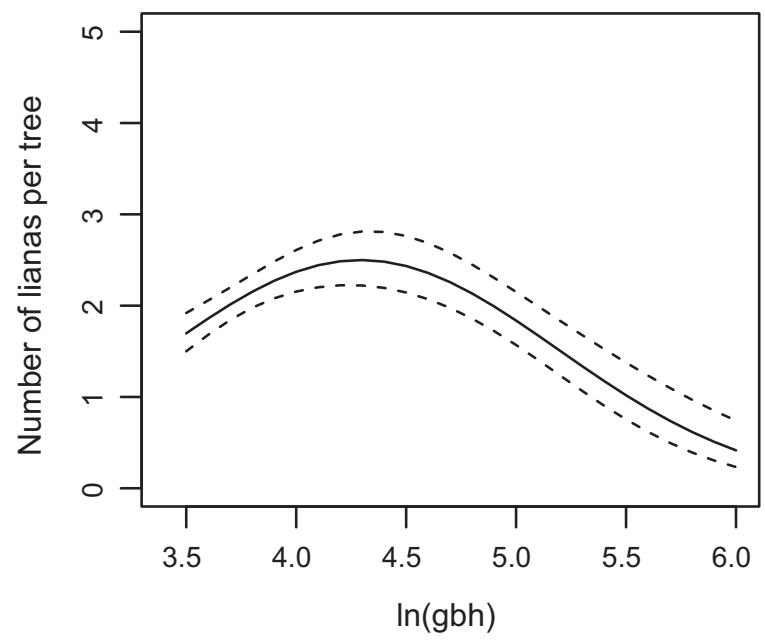

(b) 2018 MP1

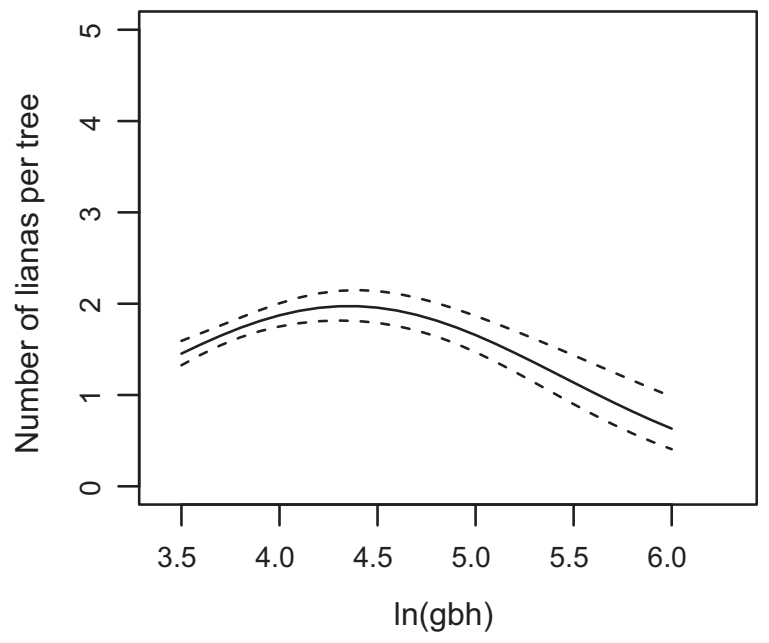

(d) 2018 MP2

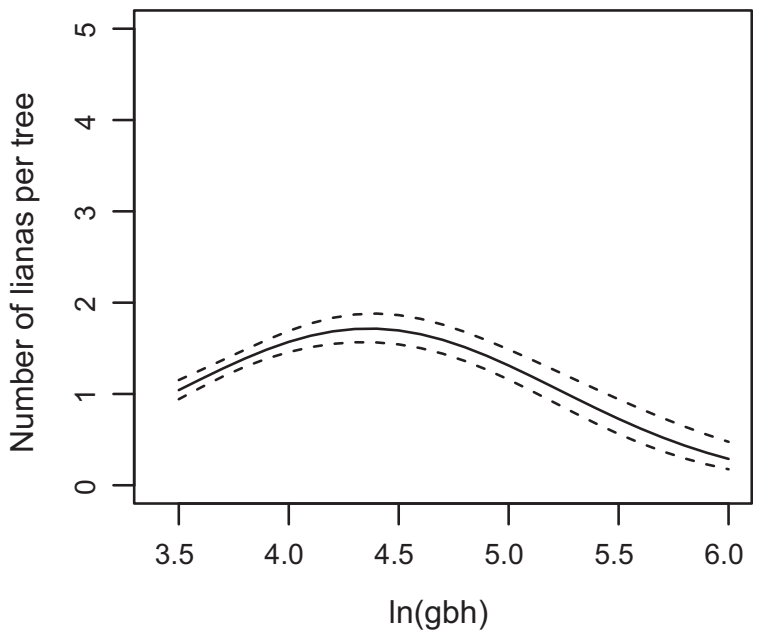

Fig. 3. Fitted relationships from negative binomial GLM regressions for the number of lianas per tree vs. tree girth at breast height size (as $\ln [\mathrm{gbh}]$ ) in main plots 1 and 2, at each census date (solid lines), with 95\% confidence bands (dashed lines).

associated with the $\ln (\mathrm{gbh})$ and $[\ln (\mathrm{gbh})]^{2}$ coefficients will be similar. The high random component (meaning a large between-tree variance) in the MP1-1988 model might have been responsible for the less pronounced quadratic fit (Appendix S1: Fig. S2).

Of the eight combinations of plot $x$ census $x$ family, two showed significance for the $\ln (g b h)$ and $[\ln (\mathrm{gbh})]^{2}$ terms: Dipterocarpaceae in MP2 for 1988 and Euphorbiaceae in MP1 for 2018 (Appendix S2: Table S1c). Accounting for spatial autocorrelation (HGLM) affected the coefficients only very slightly but the significances more, being lower for the Euphorbiaceae (remaining at $P \leq 0.05)$. Elevation was otherwise also significant for two other combinations $(P \leq 0.05)$. Trees at the family levels were less dense than overall, so the influence of spatial autocorrelation was less important.

\section{Tree survival}

Tree survival was significantly negatively related to number of lianas in both plots, although weakly with gbh and inconsistently 
Table 2. Regressions of numbers of lianas per tree on tree girth at breast height (gbh) and elevation (HGLM, quasi-Poisson error) at the censuses of 1988 and 2018, with a term, rho, for spatial autocorrelation (SAR $\dagger$ ).

\begin{tabular}{|c|c|c|c|c|}
\hline \multirow[b]{2}{*}{ Term } & \multicolumn{2}{|c|}{ MP1 } & \multicolumn{2}{|c|}{ MP2 } \\
\hline & Est \pm SE & $t$ & Est \pm SE & $t$ \\
\hline \multicolumn{5}{|l|}{1988} \\
\hline Intercept & $-2.493 \pm 1.451$ & $-1.719^{\S}$ & $-9.938 \pm 1.843$ & $-5.391^{* * *}$ \\
\hline $\ln (\mathrm{gbh})$ & $1.365 \pm 0.668$ & $2.043^{*}$ & $5.097 \pm 0.867$ & $5.877^{* * *}$ \\
\hline $\ln (g b h)^{2}$ & $-0.1284 \pm 0.0762$ & $-1.684^{\S}$ & $-0.5987 \pm 0.1007$ & $-5.944^{* * *}$ \\
\hline Elevation & $-0.01512 \pm 0.00676$ & $-2.238^{*}$ & $-0.01218 \pm 0.00399$ & $-3.051^{* *}$ \\
\hline \multicolumn{5}{|l|}{2018} \\
\hline Intercept & $-6.825 \pm 1.480$ & $-4.612^{* * *}$ & $-11.620 \pm 1.615$ & $-7.195^{* * *}$ \\
\hline $\ln (\mathrm{gbh})$ & $3.540 \pm 0.695$ & $5.097^{* * *}$ & $5.669 \pm 0.761$ & $7.453^{* * *}$ \\
\hline $\ln (g b h)^{2}$ & $-0.4092 \pm 0.0808$ & $-5.064^{* * *}$ & $-0.6477 \pm 0.0885$ & $-7.316^{* * *}$ \\
\hline Elevation & $-0.01041 \pm 0.00349$ & $-2.981^{* *}$ & $-0.01541 \pm 0.00247$ & $-6.248^{* * *}$ \\
\hline
\end{tabular}

$\dagger$ rho: $\mathrm{MP}_{88}, 0.0943 ; \mathrm{MP}_{18}, 0.0381 ; \mathrm{MP}_{28}, 0.1224 ; \mathrm{MP}_{18}, 0.0604$.

$\ddagger$ In 2018, two observations with high leverage omitted.

${ }_{* * *} P \leq 0.001 ;{ }^{* *} P \leq 0.01 ;{ }^{*} P \leq 0.05 ; \S P \leq 0.10$.

(positively) with elevation in MP2 (Table 3). Including a spatial correlation term (in the autologistic regression) changed the slope of survival on number of lianas very little (Appendix S2: Table S2a), even though the spatial covariate was significant for MP1 (Table 3). The coefficient for gbh was likewise minimally affected. Stratified random sampling gave average slopes slightly smaller than those for the autologistic fit, especially for the number of lianas term in MP1 (Appendix S2: Table S2b). Since number of lianas in the regression was those recorded in 1988, it is reasonable to conclude that increasing liana loads reduced tree survival in the $30 \mathrm{yr}$. Despite the clear trend, the pseudo- $R^{2}$ values were very low, $0.8 \%$ and $1.5 \%$ in MP1 and MP2, respectively. At the family level, survival was significantly negatively dependent on number of lianas for euphorbs in both plots $(P \leq 0.05)$ and dipterocarps in MP2 $(P \leq 0.01)$, although for MP1 the slope was in the same direction (Appendix S2: Tables S2c, d). Interestingly, the slopes of all four relationships are higher than those for all species in the plots (Table 3). What the liana loads were later after 1988 on trees that died by 2018 is unknown.

\section{Tree growth}

The negative effects of number of lianas on tree relative growth rate $\left(\mathrm{rgr}_{88-18}\right)$ were highly significant $(P \leq 0.001)$. Elevation was nonsignificant, but gbh more significant (also negative) than number of lianas (Table 4). Coefficients were very similar for MP1 and MP2. Accounting for spatial autocorrelation led to scarcely any change in coefficient values, for either the two regression approaches or stratified random sampling, indicating that local spatial dependencies were very weak (Appendix S2: Tables S3a-c). Nevertheless, testing for autocorrelation avoided type I errors.

Table 3. Autologistic regressions of tree survival 1988-2018 on number of lianas per tree (no. lianas), tree girth at breast height (gbh) in 1988, and elevation (GLM, binomial error), accounting for spatial autocorrelation (covariate).

\begin{tabular}{|c|c|c|c|c|}
\hline \multirow[b]{2}{*}{ Term } & \multicolumn{2}{|c|}{ MP1 } & \multicolumn{2}{|c|}{ MP2 } \\
\hline & Est \pm SE & $t$ & Est \pm SE & $t$ \\
\hline Intercept & $-0.834 \pm 0.380$ & $-2.20^{*}$ & $-0.434 \pm 0.385$ & $-1.13^{\mathrm{ns}}$ \\
\hline No. lianas ${ }^{1 / 2}$ & $-0.1454 \pm 0.0482$ & $-3.02^{* *}$ & $-0.1947 \pm 0.0482$ & $-4.40^{* * *}$ \\
\hline $\ln (g b h)$ & $0.2035 \pm 0.0914$ & $2.23^{*}$ & $0.1583 \pm 0.0901$ & $1.76^{\mathrm{ns}}$ \\
\hline Elevation & $-0.0024 \pm 0.0061$ & $-0.39^{\text {ns }}$ & $-0.0120 \pm 0.0044$ & $-2.74^{* *}$ \\
\hline Covariate & $0.0638 \pm 0.0309$ & $2.06^{*}$ & $0.0678 \pm 0.0454$ & $1.49^{\mathrm{ns}}$ \\
\hline
\end{tabular}

*** $P \leq 0.001 ;{ }^{* *} P \leq 0.01 ;{ }^{*} P \leq 0.05 ; \mathrm{ns}, P>0.10$. 
Table 4. General least squares regressions of tree relative growth rate (rgr) 1988-2018 on number of lianas per tree (no. lianas) and tree girth at breast height (gbh) in 1988 (mixed model, Gaussian error), accounting for spatial autocorrelation (corExp variogram).

\begin{tabular}{|c|c|c|c|c|}
\hline \multirow[b]{2}{*}{ Term } & \multicolumn{2}{|c|}{ MP1 } & \multicolumn{2}{|c|}{ MP2 } \\
\hline & Est $\pm S E$ & $t$ & Est \pm SE & $t$ \\
\hline Intercept & $0.747 \pm 0.071$ & $10.544^{* * *}$ & $0.603 \pm 0.060$ & $10.047^{* * *}$ \\
\hline No. lianas ${ }^{1 / 2}$ & $-0.0369 \pm 0.0095$ & $-3.878^{* * *}$ & $-0.0353 \pm 0.0083$ & $-4.261^{* * *}$ \\
\hline $\ln (\mathrm{gbh})$ & $-0.0998 \pm 0.0170$ & $-5.875^{* * *}$ & $-0.0755 \pm 0.0143$ & $-5.288^{* * *}$ \\
\hline
\end{tabular}

The pseudo- $R^{2}$ values for growth models were much higher than for survival, $22.5 \%$ and $32.0 \%$ for MP1 and MP2.

Simple negative binomial regressions of number of lianas in 1988 on rgr had slopes of $-0.574 \pm 0.201 \quad(z=-2.853, \quad P \leq 0.01) \quad$ and $-0.742 \pm 0.220(z=-3.376, P \leq 0.001)$ for MP1 and MP2. Repeating for number of lianas now in 2018 on rgr, the corresponding slopes were $-0.577 \pm 0.159 \quad(z=-3.643, \quad P \leq 0.001) \quad$ and $-0.508 \pm 0.172(z=-2.958, P \leq 0.01)$. Regressing the number of lianas in 1988, with a fuller model that included $\ln (\mathrm{gbh})$ and $[\ln (\mathrm{gbh})]^{2}$ of 1988, and elevation, the coefficient for $\mathrm{rgr}$ decreased for MP1 and increased moderately for MP2 (Appendix S2: Table S4). A similar regression with gbh of 2018 instead led to the rgr coefficients increasing much more for both plots, so that by partialing out the effects of gbh, the negative dependence of liana load on rgr increased. Lastly, the change in number of lianas between 1988 and 2018, treated as a Poisson variable $(n+22-$ to make all changes positive), was not significantly related to $\mathrm{rgr}$ in $\mathrm{MP1}$ $(-0.006 \pm 0.028, z=0.234, P=0.82)$ and in MP2 $(0.024 \pm 0.027, z=0.879, P=0.38)$. Adding $\ln$ (gbh) and elevation terms did not improve the fitting overall or significance of rgr.

Linear regression of rgr on number of lianas and $\ln (g b h)$ at the family level showed the expected dependence on size for the dipterocarps but not the euphorbs (Appendix S2: Table S3d, e), yet largely no or weak significance of dependence on number of lianas. The use of the variogrambased spatial autocorrelation adjustment scarcely affected the results: with coefficients increasing slightly, and for euphorbs in MP2 a small rise in significance (still only $P \leq 0.05$ ).

\section{Changes in liana abundance - contingency analysis}

Using all trees at both census dates, there was a small significant $(P<0.01)$ increase of almost $5 \%$ in the proportion of trees with lianas between 1988 and 2018 for MP1 but not MP2 where it was less at $\sim 2.5 \%$ (Table 5 ). At the family level, there

Table 5. Changes in the proportion of liana-infested trees between censuses using Pearson's $\chi^{2}$ test on all the trees and based on the randomized subsampling $\left(N^{\prime}=500\right)$.

\begin{tabular}{|c|c|c|c|c|c|c|}
\hline \multirow[b]{2}{*}{ Family } & \multicolumn{2}{|c|}{ All trees } & \multicolumn{2}{|c|}{ Randomization } & \multicolumn{2}{|c|}{ Survivors (McNemar) } \\
\hline & $\Delta$ prop. $(\%) \pm 95$ CI & $\chi^{2}$ & Mean $\chi^{2}$ & $80 \% \chi^{2}$ & $\Delta$ prop. (\%) & $\chi^{2}$ \\
\hline \multicolumn{7}{|l|}{ MP1 } \\
\hline All trees & $+4.7 \pm 3.306$ & $7.861^{* *}$ & $4.210^{*}, \dagger$ & $1.611^{\mathrm{ns}}$ & +8.9 & $15.890^{* * *}$ \\
\hline Dipterocarps & $-5.8 \pm 9.131$ & $1.493^{\mathrm{ns}}$ & $1.136^{\mathrm{ns}}$ & $0.076^{\text {ns }}$ & -8.5 & $2.161^{\mathrm{ns}}$ \\
\hline Euphorbs & $+4.3 \pm 9.777$ & $0.669^{\text {ns }}$ & $0.674^{\text {ns }}$ & $0.012^{\text {ns }}$ & +22.2 & $6.050^{*}$ \\
\hline \multicolumn{7}{|l|}{ MP2 } \\
\hline All trees & $+2.4 \pm 3.358$ & $1.852^{\mathrm{ns}}$ & $1.484^{\mathrm{ns}}$ & $0.189^{\text {ns }}$ & +7.5 & $13.499^{* * *}$ \\
\hline Dipterocarps & $+0.1 \pm 7.396$ & $<0.001^{\mathrm{ns}}$ & $0.410^{\mathrm{ns}}$ & $0.008^{\text {ns }}$ & 0.0 & $0.000^{\mathrm{ns}}$ \\
\hline Euphorbs & $+0.3 \pm 9.649$ & $<0.001^{\mathrm{ns}}$ & $0.425^{\mathrm{ns}}$ & $0.001^{\text {ns }}$ & +2.9 & $0.098^{\text {ns }}$ \\
\hline
\end{tabular}

Notes: The mean and 80th percentile of $\chi^{2}$ values are given for the randomizations. McNemar's tests were calculated using only the survivors. All tests had $1 \mathrm{df}$.

Median (range): $3.70(0.0-18.6)$.

\$ Median (range): 0.95 (0.0-10.4).

${ }_{* * *} P \leq 0.001 ;{ }^{* *} P \leq 0.01 ;{ }^{*} P \leq 0.05 ;$ ns $P>0.10$. 
were no clear significant differences, largely because sample sizes were much smaller than for all plot trees. The randomization testing supported the result for MP1 but only at $P<0.05$, and 80 percentile values were all insignificant (Table 5). Here, a qualification is needed: $\chi^{2}$ values were positively skewed, more so in MP2 than in MP1, so that the medians were lower than the means. This made the significance for all trees in MP1 possibly more marginal. Considering just the survivors, the change in MP1 and also in MP2 was larger with 8-9\% increases over time, and more strongly significant $(P<0.001)$ than when deaths after 1988 and recruits in 2018 were involved. Between families, there was pronounced difference in MP1: with a strong decrease in proportion of trees in the Dipterocarpaceae (not quite significant, however) counterbalanced by a much larger increase for the Euphorbiaceae $(\sim 22 \%)$. These differences roughly mirror the smaller relative changes at the all-trees level. However, for MP2 dipterocarps did not alter proportions and euphorbs increased just slightly (Table 5). Therefore, over all trees the proportion with lianas increased slightly in the $30 \mathrm{yr}$, more so for just survivors, but mostly for surviving euphorbs in MP1.

Considering counts of lianas per tree is more informative. Taking all trees (i.e., all families), and the single $\chi^{2}$ tests with no randomized subsampling, very highly significant differences $(P<0.001)$ in the relative frequency distributions between dates are highlighted for both plots (Table 6, Fig. 1). The large $\chi^{2}$ values in general were close to normally distributed, and so, the medians were also almost the same as the means. Not only do the randomization tests support the simpler ones, but also so do McNemar's symmetry tests for just survivors. Fig. 1 shows clearly the change in class frequencies with a decline in numbers of trees with very high loads $(\geq 8$ lianas per tree), and an increase in trees with 1-3 lianas per tree. Considering the families separately, the randomization tests did not hold up the confidence in the single overall $\chi^{2}$ test (the latter significant at $P<0.05$ in both plots, Table 6) for the dipterocarps. Conversely, while the Euphorbiaceae showed no evidence of differing class proportions in MP1, they did in MP2, supported by the randomization tests $(P<0.01)$.

The failure to detect significance in the dipterocarps under the randomization testing may have been because there were few trees in very large tree size class, the one with the high liana loads especially in 1988, and hence sample variances increased when taking only half of the trees at each census. Fig. 1c, d does not show large differences within classes, especially the case for MP1. However, coming to the Euphorbiaceae (Fig. 1e, f) there is a marked difference between dates within classes, and especially for MP2 where the frequency classes 6,7 , and $\geq 8$ are empty in 2018 (cf. the numbers in 1988), and the increases in classes 1-3 similar to the overall plot-level frequencies. Evidently, the changes in liana abundance between 1988 and 2018, while minimal in terms of proportions of trees with any lianas and none, are happening in the

Table 6. Changes in the frequency of trees in liana density classes between censuses using Pearson's $\chi^{2}$ test on all the trees and based on the randomized subsampling $\left(N^{\prime}=500\right)$.

\begin{tabular}{|c|c|c|c|c|c|c|c|}
\hline \multirow[b]{2}{*}{ Family } & \multicolumn{2}{|c|}{ All trees } & \multicolumn{3}{|c|}{ Randomization } & \multicolumn{2}{|c|}{$\begin{array}{l}\text { Survivors } \\
\text { (McNemar) }\end{array}$} \\
\hline & $\mathrm{df}$ & $\chi^{2}$ & $\mathrm{df}$ & Mean $\chi^{2}$ & $80 \% \chi^{2}$ & $\mathrm{df}$ & $\chi^{2}$ \\
\hline \multicolumn{8}{|l|}{ MP1 } \\
\hline All trees & 9 & $101.981^{* * *}$ & 8 & $53.015^{* * *}$ & $45.245^{* * *}$ & 15 & $43.9^{* * *}$ \\
\hline Dipterocarps & 6 & $14.270^{*}$ & 4 & $7.016^{\mathrm{ns}}$ & $3.879^{\text {ns }}$ & & \\
\hline Euphorbs & 7 & $9.441^{\mathrm{ns}}$ & 4 & $4.159^{\text {ns }}$ & $2.062^{\text {ns }}$ & & \\
\hline \multicolumn{8}{|l|}{ MP2 } \\
\hline All trees & 10 & $134.211^{* * *}$ & 8 & $68.690^{* * *}$ & $59.357^{* * *}$ & 15 & $67.515^{* * *}$ \\
\hline Dipterocarps & 6 & $14.267^{*}$ & 4 & $7.668^{\mathrm{ns}}$ & $4.518^{\mathrm{ns}}$ & & \\
\hline Euphorbs & 5 & $41.228^{* * *}$ & 4 & $16.304^{*}$ & $11.907^{*}$ & & \\
\hline
\end{tabular}

Notes: The mean and 80th percentile of $\chi^{2}$ values are given for the randomizations. McNemar's tests were calculated using only the survivors. Sample sizes for individual family McNemar's tests were too small.

${ }_{* * *} P \leq 0.001 ;{ }^{* *} P \leq 0.01 ;{ }^{*} P \leq 0.05 ;$ ns $P>0.10$. 
redistribution of the frequency classes: fewer to no trees with very many lianas and more trees with a few lianas by 2018. That trend was happening more strongly in MP2 than in MP1, and on account largely of changes within the Euphorbiaceae.

\section{Changes in liana abundance-regression analysis}

Negative binomial GLM regressions using the randomized subsamples involved four models, and for all trees, each of them is shown in Table 7a. Model 3, with three terms (aside from the intercept), is interesting because, for MP1, year, size, and their interaction were all significant. Without the interaction term, year became more significant but size less so, though more noticeable was that between models 2 and 3 the sign of the coefficient for year changed from negative to positive and accordingly trends with ln (gbh) were catered for more in the latter by the interaction term. For MP2, it was just year that had a consistent and strong (negative) effect. Model 3 was expanded to model 4 to have two further terms, $[\ln (\mathrm{gbh})]^{2}$ and its interaction with year, to accommodate the quadratic element of the relationship between nlianas and $\ln (\mathrm{gbh})$ and thus the different shapes of the curves between years, particularly for MP1. Even with more terms in model 4, than the other models, individual terms were all less significant for MP1, but for MP2 $\ln (\mathrm{gbh})$ and $[\ln (\mathrm{gbh})]^{2}$ featured strongly (Table 7a). It is important to recall that model 4 was more a descriptive than hypothesis testing model.

Model 4 was overall the best-fitting model for MP1 and MP2 but for slightly differing reasons. Averaged over the 500 iterations, of all four models, AIC was lowest in model 4 for both plots, and model 4 reached highest pseudo- $R^{2}$ values in MP1 and MP2 $(2.64 \%$ and $4.56 \%$, respectively;

Table 7. Negative binomial regression of number of lianas per tree on census date (year, as a factor) and tree girth at breast height (as $\ln [\mathrm{gbh}]$ ) for (a) all trees (all four models), and those in the families (b) Dipterocarpaceae and (c) Euphorbiaceae (model 1 only).

\begin{tabular}{|c|c|c|c|c|c|}
\hline \multirow[b]{2}{*}{ Model } & \multirow[b]{2}{*}{ Term } & \multicolumn{2}{|c|}{ MP1 } & \multicolumn{2}{|c|}{ MP2 } \\
\hline & & Est $\pm \mathrm{SE}$ & $z$ & Est $\pm \mathrm{SE}$ & $z$ \\
\hline \multicolumn{6}{|l|}{ (a) All } \\
\hline \multirow[t]{2}{*}{1} & Intercept & $0.771 \pm 0.044$ & $17.422^{* * *}$ & $0.701 \pm 0.049$ & $14.258^{* * *}$ \\
\hline & Year $_{18}$ & $-0.249 \pm 0.064$ & $-3.901^{* * *}$ & $-0.412 \pm 0.070$ & $-5.903^{* * *}$ \\
\hline \multirow[t]{3}{*}{2} & Intercept & $0.040 \pm 0.237$ & $0.169^{\text {ns }}$ & $0.596 \pm 0.254$ & $2.348^{*}$ \\
\hline & Year $_{18}$ & $-0.240 \pm 0.064$ & $-3.774^{* * *}$ & $-0.413 \pm 0.070$ & $-5.913^{* * *}$ \\
\hline & $\ln [g b h]$ & $0.180 \pm 0.057$ & $3.112^{* *}$ & $0.026 \pm 0.062$ & $0.425^{\mathrm{ns}}$ \\
\hline \multirow[t]{4}{*}{3} & Intercept & $-0.492 \pm 0.327$ & $-1.502^{\mathrm{ns}}$ & $0.831 \pm 0.356$ & $2.337^{*}$ \\
\hline & Year $_{18}$ & $0.930 \pm 0.471$ & $1.974^{*}$ & $-0.874 \pm 0.503$ & $-1.735^{*}$ \\
\hline & $\ln [\mathrm{gbh}]$ & $0.310 \pm 0.080$ & $3.874^{* * *}$ & $-0.032 \pm 0.087$ & $-0.368^{\mathrm{ns}}$ \\
\hline & Year $_{18}: \ln [\mathrm{gbh}]$ & $-0.290 \pm 0.116$ & $-2.508^{*}$ & $0.114 \pm 0.123$ & $0.924^{\mathrm{ns}}$ \\
\hline \multirow[t]{6}{*}{4} & Intercept & $-3.778 \pm 2.095$ & $-1.781^{\S}$ & $-10.756 \pm 2.543$ & $-4.209^{* * *}$ \\
\hline & Year $_{18}$ & $-3.636 \pm 3.110$ & $-1.168^{\mathrm{ns}}$ & $-1.681 \pm 3.661$ & $-0.464 \mathrm{~ns}$ \\
\hline & $\ln [\mathrm{gbh}]$ & $1.862 \pm 0.973$ & $1.888^{\S}$ & $5.449 \pm 1.192$ & $4.549^{* * *}$ \\
\hline & Year $_{18}: \ln [\mathrm{gbh}]$ & $1.860 \pm 1.451$ & $1.281^{\mathrm{ns}}$ & $0.506 \pm 1.718$ & $0.300^{\mathrm{ns}}$ \\
\hline & $(\ln [\mathrm{gbh}])^{2}$ & $-0.180 \pm 0.111$ & $-1.584^{\mathrm{ns}}$ & $-0.636 \pm 0.138$ & $-4.597^{* * *}$ \\
\hline & Year $_{18}:(\ln [\mathrm{gbh}])^{2}$ & $-0.248 \pm 0.167$ & $-1.488^{\text {ns }}$ & $-0.047 \pm 0.199$ & $-0.244^{\mathrm{ns}}$ \\
\hline \multicolumn{6}{|l|}{ (b) Dipt } \\
\hline \multirow[t]{2}{*}{1} & Intercept & $0.599 \pm 0.161$ & $3.747^{* * *}$ & $0.302 \pm 0.155$ & $1.959^{*}$ \\
\hline & Year & $-0.607 \pm 0.234$ & $-2.589^{* *}$ & $-0.481 \pm 0.221$ & $-2.172^{*}$ \\
\hline \multicolumn{6}{|l|}{ (c) Euph } \\
\hline \multirow[t]{2}{*}{1} & Intercept & $0.648 \pm 0.116$ & $5.590^{* * *}$ & $0.687 \pm 0.137$ & $5.035^{* * *}$ \\
\hline & Year & $-0.019 \pm 0.168$ & $-0.116^{\mathrm{ns}}$ & $-0.647 \pm 0.202$ & $-3.207^{* *}$ \\
\hline
\end{tabular}

Notes: The four models are follows: 1, nlianas $\sim$ year; 2, nlianas $\sim$ year $+\ln [\mathrm{gbh}] ; 3$, nlianas $\sim$ year $+\ln$ [gbh] + year: $\ln$ [gbh]; and 4, nlianas $\sim$ year $+\ln [\mathrm{gbh}]+$ year: $\ln [\mathrm{gbh}]+(\ln [\mathrm{gbh}])^{2}+$ year: $(\ln [\mathrm{gbh}])^{2}$. The values are averages of $N^{\prime}=500 \mathrm{ran}-$ domizations.

${ }_{* * *} P \leq 0.001 ;{ }^{* *} P \leq 0.01 ;{ }^{*} P \leq 0.05 ; \S P \leq 0.10 ;$ ns $P>0.10$. 
Appendix S3: Table S1). Highest LRs were attained when model 4 was compared to model 1 , again in both plots $\left(\mathrm{LR}_{03}=32.3\right.$ and 46.4); the changes from models 1 to 3 were nearly as significant $\left(\mathrm{LR}_{13}=22.7\right.$ and 45.8$)$. The six paired comparisons were the models coded 12, 13, 14, 23, 24, and 34. Notable was that LRs for models 1-2 in both plots were relatively low (Appendix S3: Table S1), indicating that model fitting markedly improved when interactions between year and $\ln$ $(\mathrm{gbh})$ and $[\ln (\mathrm{gbh})]^{2}$ were estimated. Probabilities of the $\chi^{2}$ change in deviance when comparing models were lowest for model 1 vs. model 4 in MP1, and even lower for models 1, 2, and 3 with model 4 in MP2.

Considering the frequencies with which models were selected across the iterations, lowest AIC values were attained with model 4 in 497 of 500 iterations for MP1 (model 3 in three), and all 500 for MP2. Likewise, model 4 had the highest LR values among all six comparisons in all 500 for both plots, and similarly, pseudo- $R^{2}$ was highest for all 500 per plot. Such consistency is not surprising given the small SEs of the statistics (Appendix S3: Table S1). Delta-AIC was found for models paired in the same way done for LR, and here, 475 of 500 were largest for model 1 minus model 4 for MP1, but 373 of 500 for model 3 minus model 4 in MP2 (Appendix S3: Table S2). Similarly, the significance of the $\chi^{2}$ measure of deviance change was highest for 455 or 500 iterations in MP1 for the 14 comparison and 438 or 500 in MP2 for the 34 one. LR was never highest for the model 23 comparison or were the corresponding probabilities of the deviance change the lowest; very rarely were these statistics the highest, respectively, and lowest for model comparisons 12 and 13 either (Appendix S3: Table S2). These last results point to an important difference between the plots: While model 4 was overall best for both, the most improvement for MP1 was between models 1 and 4, but for MP2 it was between models 3 and 4 .

In these glm.nb fits, year was coded as a factor. This meant that year 18 was the difference from the reference year 88 , and likewise, interactions between $\ln (\mathrm{gbh})$ and $[\ln (\mathrm{gbh})]^{2}$ with year were differences in their corresponding slopes (Appendix S2: Table S4). For MP1, nlianas in 2018 became a decreasing proportion of the nlianas in 1988 as gbh increased, going from 85\% at $\ln (\mathrm{gbh})=3.5(\mathrm{gbh}=33.1 \mathrm{~cm})$ to $40 \%$ at $\ln$ $(\mathrm{gbh})=5.5 \quad(\mathrm{gbh}=244.7 \mathrm{~cm}) \quad($ Appendix $\quad \mathrm{S} 2:$ Table S5a). But for MP2, the change was very different, from $62 \%$ rising to $73 \%$ for the same gbh range (Appendix S2: Table S5b). So, while model 4 was the better one for MP1 (cf. models 1-3) and showed the strongest change with respect to gbh, its main terms became less, and the interaction terms conversely more, significant.

Model 4 was also run on the full data, that is, all trees without random subsampling at the dates, and the coefficients fitted matched the averages from the randomizations very well. (Appendix S2: Table S6a). Further, the coefficients from model 4 could be used to reconstruct the individual equations for the dependence of nlianas on $\ln (\mathrm{gbh})$ and $[\ln (\mathrm{gbh})]^{2}$ at each date (similar to those reported in Table 2 without the elevation term, again matching closely) (Appendix S2: Table S6b). This demonstrated that in testing for change in nlianas over time (the year factor) the gbh curves were being modeled by the interaction terms correctly. Predicted (marginal) means of nlianas in 1988, and in 2018 - using a mean $\ln (\mathrm{gbh})$ and $[\ln (\mathrm{gbh})]^{2}$ common to years - were very similar across the four models, with just small decreases of 1.5$1.8 \%$ for MP1, and 2.8-3.0\% in MP2, between models 1 and 4 (Appendix S2: Table S7a). Predicted means at the family level were also very similar (Appendix S2: Table S7b). The means resulting from models 1 and 2 are, naturally, virtually the same as the values given in Table 1.

\section{Liana abundance and dynamic status}

Among the trees that died 1988-2018, a higher proportion had lianas present in 1988 compared with those that survived (Table 8). This was especially significant in MP2. Correspondingly, dead trees had on average more lianas per tree than survivors. Trees in both the Dipterocarpaceae and Euphorbiaceae followed the same trends, again more strongly in MP2 than in MP1. Survivors of dipterocarps had less than half the numbers per tree than those that died. Recruits on the other hand by 2018 had proportionally fewer trees with lianas than survivors, and less lianas per tree, especially in MP1 (Table 9). Differences were less strong at the family level, 
Table 8. The proportions of trees with lianas for those trees that survived vs. died $\left(n_{\mathrm{s}}, n_{\mathrm{d}}\right)$ between censuses 1 and $2(1988,2018)$, and their corresponding mean numbers per tree, in the two plots for all families (all trees) and for those trees in the Dipterocarpaceae (Dipt) and Euphorbiaceae (Euph).

\begin{tabular}{|c|c|c|c|c|c|c|c|c|c|}
\hline \multirow[b]{2}{*}{ Family } & \multirow[b]{2}{*}{ Plot } & \multicolumn{2}{|c|}{ No. trees } & \multicolumn{3}{|c|}{ Percentage of trees with lianas } & \multicolumn{3}{|c|}{ Mean no. lianas/tree } \\
\hline & & Died & Survived & $\overline{\text { Died }}$ & Survived & $\chi^{2} \dagger$ & Died & Survived & $z \dagger$ \\
\hline \multirow[t]{2}{*}{ All } & MP1 & 926 & 778 & 62.7 & 57.3 & $4.96^{*}$ & 2.326 & 1.972 & $-2.367^{*}$ \\
\hline & MP2 & 716 & 943 & 59.6 & 50.3 & $14.03^{* * *}$ & 2.402 & 1.725 & $-4.238^{* * *}$ \\
\hline \multirow[t]{2}{*}{ Dipt } & MP1 & 105 & 141 & 50.5 & 42.6 & $1.22^{\mathrm{ns}}$ & 2.114 & 1.638 & $-1.059^{\mathrm{ns}}$ \\
\hline & MP2 & 133 & 197 & 44.4 & 31.5 & $5.14^{*}$ & 2.000 & 0.929 & $-3.215^{* *}$ \\
\hline \multirow[t]{2}{*}{ Euph } & MP1 & 152 & 54 & 69.7 & 50.0 & $5.95^{*}$ & 2.112 & 1.389 & $-2.078^{*}$ \\
\hline & MP2 & 114 & 105 & 61.4 & 43.8 & $6.10^{*}$ & 2.509 & 1.438 & $-2.538^{*}$ \\
\hline
\end{tabular}

Note: $\chi^{2}$ is from the Pearson test of association, and $z$ is the statistic from the binomial GLM regression.

$\dagger \mathrm{df}=1 ; \ddagger \mathrm{df}=1, n_{\mathrm{s}}+n_{\mathrm{d}}-2$.

*** $P \leq 0.001 ;{ }^{* *} P \leq 0.01 ;{ }^{*} P \leq 0.05$; ns $P>0.10$.

Table 9. The proportions of trees with lianas for those that survived vs. recruited $\left(n_{s}, n_{r}\right)$ between censuses 1 and $2(1988,2018)$, and their corresponding mean numbers per tree, in the two plots for all families (all trees) and for those trees in the Dipterocarpaceae (Dipt) and Euphorbiaceae (Euph).

\begin{tabular}{|c|c|c|c|c|c|c|c|c|c|}
\hline \multirow[b]{2}{*}{ Family } & \multirow[b]{2}{*}{ Plot } & \multicolumn{2}{|c|}{ No. trees } & \multicolumn{3}{|c|}{ Percentage of trees with lianas } & \multicolumn{3}{|c|}{ Mean no. lianas/tree } \\
\hline & & Recruited & Rec.-Died & Survive & Recruited & $\chi^{2} \dagger$ & Survived & Recruited & $z \dagger$ \\
\hline \multirow[t]{2}{*}{ All } & MP1 & 921 & -5 & 66.2 & 64.0 & $0.84^{\mathrm{ns}}$ & 1.923 & 1.486 & $-4.536^{* * *}$ \\
\hline & MP2 & 889 & 173 & 57.8 & 55.5 & $0.93^{\mathrm{ns}}$ & 1.408 & 1.253 & $-1.906^{\S}$ \\
\hline \multirow[t]{2}{*}{ Dipt } & MP1 & 106 & 1 & 34.0 & 48.1 & $4.42^{*}$ & 0.950 & 1.066 & $0.512^{\mathrm{ns}}$ \\
\hline & MP2 & 156 & 23 & 31.5 & 43.6 & $4.99^{*}$ & 0.716 & 1.000 & $1.692^{\S}$ \\
\hline \multirow[t]{2}{*}{ Euph } & MP1 & 136 & -16 & 72.2 & 67.6 & $0.19^{\mathrm{ns}}$ & 2.093 & 1.809 & $-0.822^{\text {ns }}$ \\
\hline & MP2 & 120 & 6 & 46.7 & 59.2 & $3.03^{\S}$ & 0.943 & 1.150 & $1.144^{\mathrm{ns}}$ \\
\hline
\end{tabular}

Notes: In comparing Tables 8 and 9, the numbers of trees surviving are the same (as in Table 8), but the numbers of trees dying and recruiting differed (column "rec-die" here). $\chi^{2}$ is from the Pearson test of association, and $z$ is the statistic from the binomial GLM regression.

$\dagger \mathrm{df}=1 ; \ddagger \mathrm{df}=1 ; n_{s}+n_{r}-2$.

${ }^{* *} P \leq 0.001 ; * P \leq 0.05 ; \S P \leq 0.10$, ns $P>0.10$.

except for dipterocarps where the proportion of trees with lianas was significantly less on survivors than on recruits in both plots, though numbers per tree were similar.

\section{Liana densities per tree for individual species}

The mean numbers of lianas per tree for the 51 and 52 more frequent tree species in MP1 and MP2 together at 1988 and 2018 were merged to 61 species in common (Appendix S4: Table S1). Mean species' number of lianas per tree was very weakly correlated with OUI $(r=-0.006, \mathrm{df}=49$, $P=0.97)$ and $\ln (\mathrm{gbh}) \quad(r=-0.047, \quad \mathrm{df}=49$, $P=0.74)$ in 1988 though slightly more strongly in $2018(r=-0.175, \mathrm{df}=50, P=0.21 ; r=-0.278$, $\mathrm{df}=50, P=0.046$ correspondingly). OUI and $\ln$ (gbh) were highly correlated in $1988(r=0.859$, df $=48, P<0.001$; with Nothaphoebe sp. dropped because its $\ln (\mathrm{gbh})$ value was very heavily outlying) and in $2018(r=0.904, \mathrm{df}=50, P<0.001)$. However, the poor correlations hide an interesting separation between six and seven canopy/ emergent dipterocarps, with high OUI values but very low numbers of lianas per tree, vs. the rest that follow a general positive trend (Figs. 4, 5). The separation is weak in 1988 but becomes distinct in 2018. Similar patterns arise when plotting against $\ln (\mathrm{gbh})$ instead of OUI. This separated group of dipterocarps clearly have very much lower numbers of lianas per tree than would be predicted from the general trend with increasing OUI of all the other species.

Species' means of numbers of lianas per tree were weakly correlated with percentages of 1988 


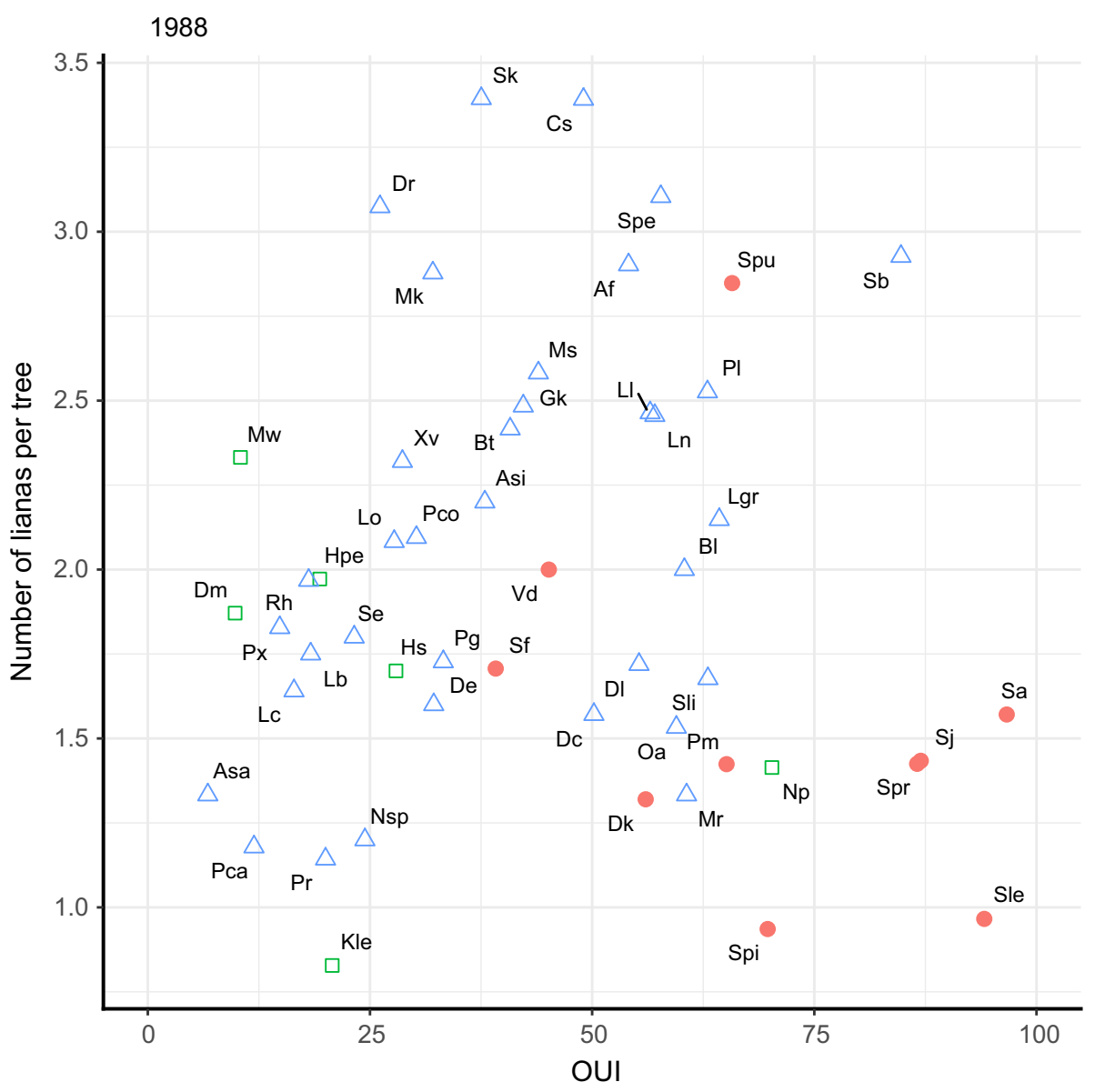

Fig. 4. Relationship between mean species' number of lianas per tree and the over-understory index (OUI), for species with $\geq 20$ trees in plots MP1 and MP2 combined in 1988. Closed red circles, Dipterocarpaceae; open green squares, Euphorbiaceae; open blue triangles, other families.

trees that died by $2018(\% \mathrm{~d})$ at both 1988 $(r=-0.103, \quad \mathrm{df}=49, \quad P=0.48) \quad$ and 2018 $(r=-0.061, \mathrm{df}=50, P=0.67)$, and also, this was the case for correlations with $\ln (\mathrm{rgr})$ of small trees in $1988(r=-0.094, \mathrm{df}=48, P=0.52$; no rgr estimate for Nothaphoebe sp.) and 2018 $(r=-0.269, \mathrm{df}=50, P=0.054)$. Separation of groups, especially of the large dipterocarps, was much less evident although the 6-7 species that featured on the OUI graphs also had among the highest rgr values when their trees are small (Appendix S4: Fig. S1).

Forty-two species occurred at both dates on the $\geq 20$-trees criterion, and their liana numbers per tree in 1988 and 2018 were positively correlated $(r=0.661, \quad \mathrm{df}=40, \quad P<0.001 ; \quad$ Fig. $)$.
Although the variables at the two dates are not fully independent from one another (they shared survivors and their lianas), the dependence of numbers in 2018 on those in 1988 is instructive even if the significance level attached is inflated due to some autocorrelation: nlianas $_{18}=0.411+0.511 \cdot$ nlianas $_{88}(F=31.1, \mathrm{df}=$ $1,40 ; P<0.001)$. On average, all species had fewer lianas in 2018 than 1988, but the reduction was most when the numbers in 1988 were higher. The fitted line shows the difference from a 1:1 expectation. The average of the plots in 1988 was 2.091 lianas per tree and 1.510 per tree in 2018 (from Table 1). Putting this average for 1988 in the fitted linear model gave an estimated mean of 1.472. An interesting feature of Fig. 6 is 


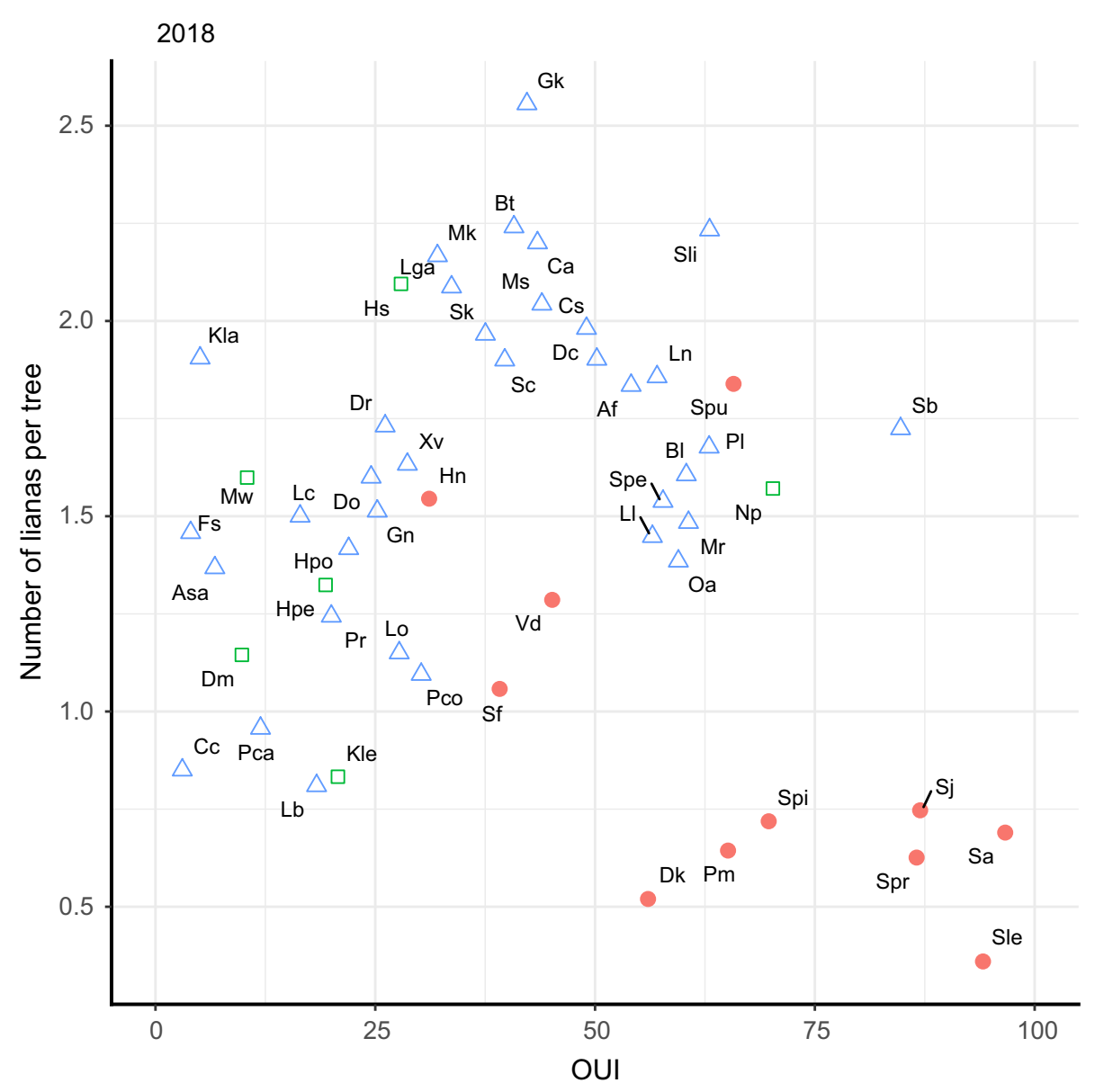

Fig. 5. Relationship between mean species' number of lianas per tree and the over-understory index (OUI), for species with $\geq 20$ trees in plots MP1 and MP2 combined in 2018. Symbols as in Fig. 4 .

that most of the dipterocarps that attain very large sizes are below the fitted line, having among the lowest means in 2018, reflecting the patterns in Fig. 5. The exception again is S. pauciflora. Differences between species' means of numbers of lianas per tree (1988- minus 2018values) were moreover also not significantly correlated with $\ln (\mathrm{rgr})$ of small trees $(r=0.093$, $\mathrm{df}=40, \quad P=0.56) \quad$ or $\quad$ with $\% \mathrm{~d} \quad(r=-0.115$, $\mathrm{df}=40, P=0.47)$. No phylogenetic adjustment was necessary since the results were at best only marginally significant. Liana density was related to species mean size $(\ln [\mathrm{gbh}])$, and form of size distribution defining story (OUI) when conditioned on tree family, that is, dipterocarps vs. non-dipterocarps, but not with the basic growth rate of their trees when small and species mortality rates.

\section{DISCUSSION}

\section{Changing liana abundance}

The two liana censuses covered a recent 30-yr period in a forest considered to be in a stage of late-successional recovery from a major natural drought disturbance, probably in the 1870s. Indicators were the very small proportion of plot area with gaps, very few pioneer stage trees, few large lying stems, no evidence of major wind damage, and total tree basal area approaching an expected site maximum accompanied by declining tree density (Newbery et al. 1992). This contrasts strongly with the majority of liana studies to date, which were conducted in heavily disturbed and secondary successional forests, or forests with a strong component of fast-growing light-demanding species (Visser et al. 2018a). 


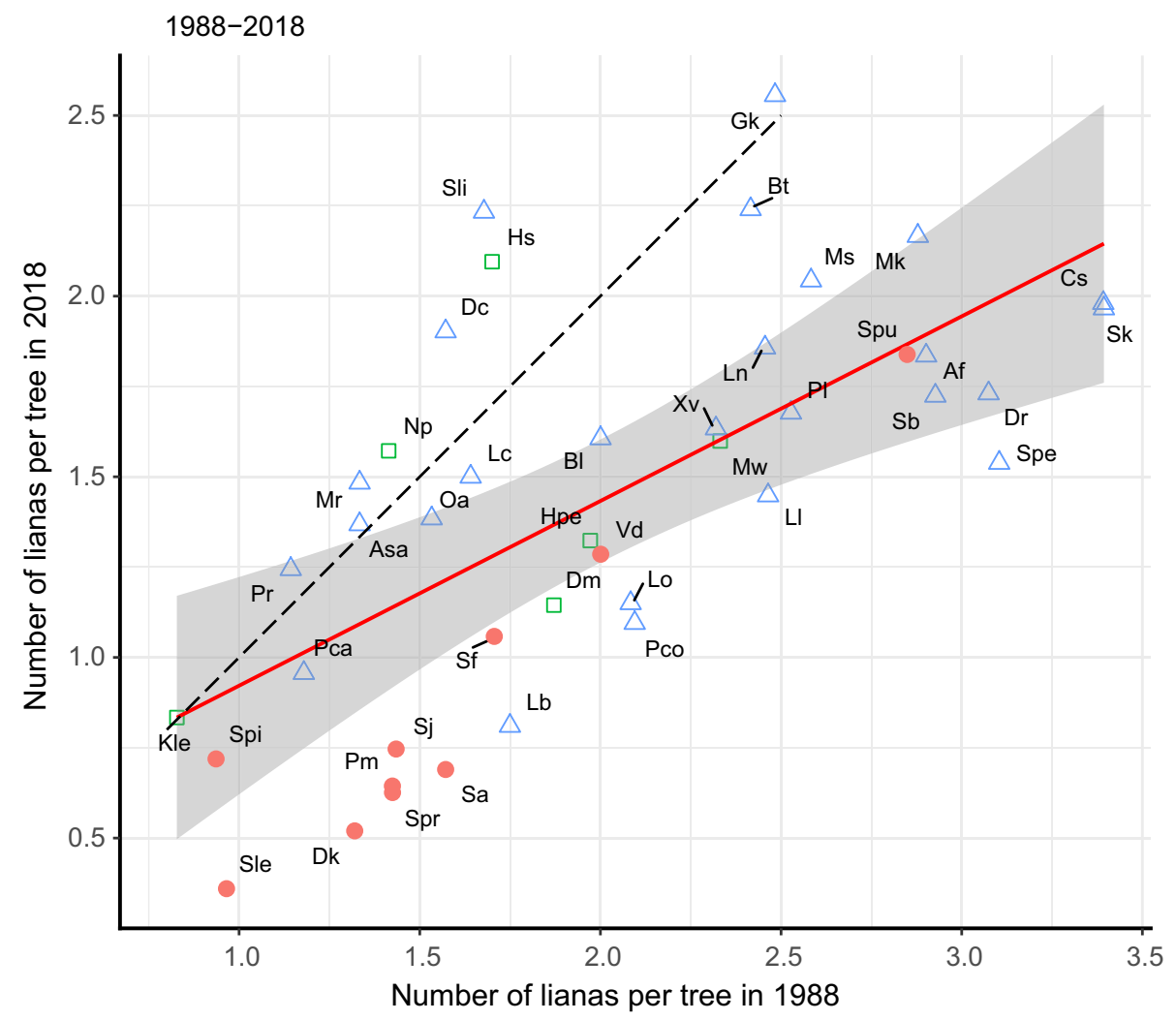

Fig. 6. Differences in species' mean numbers of lianas per tree (1988 minus 2018 values) for species with $\geq 20$ trees at both censuses, in plots MP1 and MP2 combined. Symbols as in Fig. 4. The red line is the linear fit for all points, with $99 \%$ confidence band; the black dashed line is the 1:1 no-change expectation.

The closest and most relevant comparison to the present study is that of Wright et al. (2015). At Danum, the proportion of trees $\geq 30 \mathrm{~cm}$ dbh $(95.4 \mathrm{~cm} \mathrm{gbh})$ with lianas present was $44.5 \%$ in 1988 and $48.6 \%$ in 2018 , values slightly less on average than at Pasoh (see Introduction), those at Pasoh decreasing and at Danum increasing a little. If the crown position class 'emergent' for Pasoh (Fig. 1c of Wright et al. 2015, for 2014) can be equated to the size class 4 at Danum (Fig. 3, for 2018), the corresponding proportions of the very largest trees with no lianas were $\sim 25 \%$ and $75 \%$, respectively, a threefold higher liana-free stem frequency at Danum. Further comparisons are problematic because the studies used different methods of recording liana abundance (percent canopy cover vs. numbers per stem), and had different census intervals (12 vs. $30 \mathrm{yr})$. With some approximate adjustments, the rate of decline seemed much steeper at Danum than at Pasoh.
Between the two censuses, tree size-frequency distributions and major family contributions did not change significantly, although above a gbh of $240 \mathrm{~cm}$ tree frequencies at Danum were more erratic with numbers of trees in 2018 larger than in 1988 for the two largest classes (Appendix S1: Fig. S1). The average numbers of lianas per tree decreased by $25 \%$ in the $30-y r$ period, though the proportions of trees with lianas increased by $3.5 \%$. This came about because in 2018 relatively more trees had few lianas (1-3 in number), and relatively fewer trees had many lianas $(\geq 8)$, than in 1988. Numbers of trees with no lianas remained on average very similar. The two plots differed importantly, however, in their liana dynamics. Large-sized trees had mainly higher liana loads than medium-sized and very large ones, except for MP1 in 1988 when very large trees had similarly high loads to large trees. The statistical errors were relatively high here due to the fewer trees in the very large tree size class. 
Accordingly, this latter class showed a much faster decline between the censuses in MP1 than in MP2. Number of lianas per tree decreased significantly with topographic elevation overall: They were fewer on ridges than lower slopes. Despite the often high significance of coefficients from the model fitting, total variances accounted for were low, indicating that many other unknown sources of variation besides tree size and elevation were important.

In both plots, trees with many lianas in 1988 survived significantly less well than those with none or few. Tree survival also decreased with elevation, more strongly in MP2 than in MP1, but it was unrelated to tree size. Likewise, when liana loads increased this led to significantly reduced rgr of gbh, but rgr was unaffected by elevation. Final model fits accounted for far more variance in growth ( $27 \%$ on average) than in survival $(\sim 1 \%)$. Thus, if lianas were affecting tree growth negatively, then survival would be expected to decrease as well, as reduced growth normally enhances tree mortality. Partialing out elevation still left survival and growth depending on tree size. Differences between families in the dependence of their trees' growth rates on liana load were not consistent between plots though. For dipterocarps the relationship was negative in MP2 but not so in MP1, and for euphorbs it was slightly negative both in MP1 and in MP2. The decrease in rgr with increasing tree size for all trees was highly significant, for the dipterocarps too, but not for the euphorbs, at the family level.

Monte Carlo randomization testing showed that the increase in proportions of trees with lianas was marginally significant in MP1 but not significant in MP2. For survivors alone, the changes were stronger and more significant, indicating perhaps that mortality and recruitment of trees in the period were offsetting accumulation on survivors. The most robust results, statistically, came from the randomization testing for numbers of lianas per tree, confirming the decline between 1988 and 2018 in MP1 and MP2, in the proportion of trees with high numbers of lianas per tree, and the corresponding increase in the proportion of trees with very few lianas. This form of testing, taking independent tree samples at the two census dates (i.e., samples having different survivors), also removes survival bias due to trees with heavy liana loads often dying along with their loads and the liana counts becoming zero (Visser et al. 2018a, b). This bias has, inter alia, important consequences for understanding how liana loads, or burdens, affect tree population growth rates (Muller-Landau and Vissier 2019). Randomization also avoided the more essential problem of a lack of independence caused by survivors being counted in the samples of both census dates. As to be expected for a long-lived structural parasite, liana numbers increased with the age of the surviving host unless they were shed. Even so, trees die for many other reasons, and infestation by lianas is but one contributor.

The weak, or lacking, differences in mean numbers of lianas between censuses in the Dipterocarpaceae in both plots may indicate that for this family, summed across the size classes, lianas were being gained at a similar rate to them being lost without undue mortality of large and very large trees. As discussed later, the interaction between lianas and the very largest trees points to an important process operating in this dominant tree family. Most of the large to very large trees in the Danum plots are dipterocarps (Newbery et al. 1992, 1996). For the smaller Euphorbiaceae, however, there was little difference in mean number of lianas in MP1 yet substantial decreases in MP2. The negative binomial regressions for the changes in numbers of lianas per tree, under randomization testing, furthermore highlighted the importance of the year $\times$ size interaction, which again differed between plots. The hierarchical model comparisons confirmed that largest reductions over time were for the very large trees, especially in MP1. The differing patterns in small-scale dynamics of the trees and their lianas highlight the fundamental role of location, a spatial variability between plots set in the context of larger-scale temporal change.

In this study, only the number of lianas per tree stem was recorded and not liana stem diameter or canopy crown infestation. At two other sites, these variables were found to be rather weakly correlated with one another (van der Heijden et al. 2008, Cox et al. 2019) and that might have been the case at Danum too. Good reliable observations of all parts of crowns of all trees (i.e., understory, intermediate, and overstory) from the forest floor were not feasible at Danum 
because of the high tree density and the strongly gradated forest profile. The lack of good viewing points would have meant omitting an unacceptably high proportion of the trees sampled and a resulting selective bias in cover values. The number of lianas per tree is a first approximation to liana abundance: A more quantitative measure would have been liana basal area per tree. How lianas were distributed above in trees, and indeed which ones climbed from medium to large individuals was not recorded. Putz and Chai (1987) found though that each canopy liana $(>2 \mathrm{~cm} \mathrm{dbh})$ connected on average 1.4 trees $>20 \mathrm{~cm} \mathrm{dbh}$ in primary dipterocarp forest at Lambir, Sarawak.

When considering rgr response, the causal direction is not unambiguous. On the one hand, high liana loads might have reduced rgr more than did low ones, but, on the other, trees that were growing fast may have discouraged the survival of lianas more than those growing slowly. This might be connected with branch shedding: Faster-growing trees would probably also shed branches faster than slower-growing ones. If slower-growing trees suffered from higher liana loads, they would have been more likely to die than those faster-growing ones with lower loads. When the potential for a tree to grow tall was high, the relative costs of branch shedding would be low, that is, they are affordable, compared with the converse. An important qualification to the analysis is that the rgr models were based on the growth of final survivors over the 30-yr period, and not on the rgr they attained up until the time they died on a shorter intervalwise basis using the tree census data (cf. Newbery and Ridsdale 2016).

\section{Tree-liana interactions}

Loss of lianas is closely connected with death of their hosts. Tree dynamics found using dates near to the two liana censuses can be more accurately derived by averaging over to the four periods between the main tree censuses of 1986, 1996, 2001, 2007, and 2015 (Newbery et al. 1999, 2011; D. M. Newbery, P. Stoll, and M. Lingenfelder, unpublished data). These further estimates reduce the length of interval bias inherent to mortality rate calculations (Sheil and May 1996). The first and last tree censuses were 2-3 yr prior to the liana ones and showed slightly lower differences in rates between plots compared with those based on the liana-censused trees. For all trees, MP1 had a 33-35\% higher annualized mortality rate $\left(m_{a}\right)$ than MP2 across three size classes 30 to $<60, \quad 60$ to $<120$, and $\geq 120 \mathrm{~cm}$ gbh (Appendix S1: Table S2). For medium-sized trees, this proportional difference between plots was similar for the Dipterocarpaceae, but it was $>60 \%$ for the Euphorbiaceae. Plots differed hardly at all for large and very large dipterocarps (1-8\%) but were somewhat lower for large euphorbs $(20 \%)$. Because of their life form, species in the Euphorbiaceae hardly ever reach the very large size class to allow estimates.

Between 1986 and 2015, densities of trees (N/ ha) $\geq 30 \mathrm{~cm}$ gbh changed from 484 to 452 in MP1 and 455 to 477 in MP2. The corresponding basal area abundances $\left(\mathrm{m}^{2} / \mathrm{ha}\right)$ were 26.34 to 27.14 in MP1 and 26.79 to 29.28 in MP2. These density shifts account for the differing percent changes in numbers of lianas per tree, when based on number of lianas per plot area and mean number per tree. Over the almost $30 \mathrm{yr}$, MP1 with the higher mortality, particularly of very large trees, decreased in tree density and made little gain in basal area, but MP2 with lower mortality increased in density and basal area, having more very large trees in MP2 than in MP1 (Newbery et al. 1992; D. M. Newbery, unpublished data; Appendix S1: Fig. S1). In addition, largest tree, that is maximum, heights (for $\geq 100 \mathrm{~cm} \mathrm{dbh}$, bar one at $90 \mathrm{~cm}$ ) were higher in MP2 than in MP1 by $8.1 \mathrm{~m}$ at the end of 2016 (62.1 vs. $54.0 \mathrm{~m}$, $n=19$ and 23; D. M. Newbery, unpublished data). Again, it is the important local plot-scale variation in the forest structure and dynamics that provided detailed insights into the tree-liana interactions.

Of the main census trees with $\mathrm{gbh} \geq 240 \mathrm{~cm}$ in $1986(n=89)$, the proportions dying by 2015 were $33 \%(13 / 39)$ and $48 \%(24 / 50)$ in MP1 and $\mathrm{MP2}$, respectively $\left(\chi^{2}=1.94, \mathrm{df}=1, P=0.16\right)$, or as $m_{a}$ over the $28.74 \mathrm{yr}, 1.40$ and $2.25 \% / \mathrm{yr}$. In the liana census data, the number alive at 1988 was 36 and 44, respectively, just one death in MP1 between 1986 and 1988 . These $m_{a}$ values are indeed the reverse of those for the very large trees $(\mathrm{gbh} \geq 120 \mathrm{~cm})$ with respect to plot differences. Nevertheless, the apparent trends among the very largest trees in number of lianas with increasing gbh (Fig. 3, Appendix S1: Fig. S2a, b) 
is due to three liana counts of 20, 18 (MP1), and 13 (MP2): one tree dying and two surviving. In both plots, there were only three trees with $\geq 7$ lianas each.

While tree mortality rates were higher in MP1 than in MP2 for all three size classes, it was the disproportionately higher-laden trees in the very large size class that led to more lianas being lost in MP1 than in MP2 in that size class. The large and very large trees in MP1 most likely had higher liana loads than those in MP2 even before 1988. In MP1, they had then more lianas to lose by 2018 than those in MP2 by 2018 . Without precise data on large branch fall rates, the large-tovery-large trees must be assumed to have been shedding their lianas at similar rates in MP1 and MP2. Therefore, the difference between the plots, for a majority of the dipterocarps at least, was a result of tree mortality over and above a higher branch fall loss in very large trees compared with large- and medium-sized ones.

For trees overall, to show similar changes in distributions of numbers of lianas per tree in both plots implies a different process to have been operating in MP2 from that in MP1. The main changes were in the medium-sized understory trees, where tree mortality was much higher in MP2 than in MP1. However, survivors in these size classes were also slowly accumulating lianas, perhaps as newly rooted ones or coming from fallen trees (Putz 1984). Recruits, being younger and smaller than other trees, would have had little time to accumulate lianas, and hence, their loads were relatively low by 2018 . The different dynamics in the two plots resulted in similar frequency distributions of lianas per tree though. The overstory dipterocarps seemingly have more the option of branch shedding to lose lianas, but the understory euphorbs far less (Campbell and Newbery 1993). The euphorbs can for the most part only lose their lianas on the death of the host. An interesting feature is the higher proportion of trees with no lianas in MP2 than in MP1, particularly for the dipterocarps.

If fast-growing medium-sized dipterocarps (Newbery et al. 1999), moving from under- to overstory, were losing branches, they might well have shed their lianas along with them. Shedding lianas with branches is one obvious way to relieve the load and recover rgr for onward growth for an overstory species, and thereby wincrease its survival under competition with not just the lianas but also neighboring trees. As understory species do not grow into the overstory at all, these considerations are not so important for them. Understory trees incidentally provide a means for lianas to step up onto neighboring overstory trees. If large trees remained infested in their canopies and yet have no lianas on their own stems at near-ground level, these lianas are presumably coming across from neighboring medium-sized trees. This would cause a mismatch between stem counts and liana effects.

The influence of spatial autocorrelation on the fitted values of the independent variables' coefficients was often quite small, both when the dependent variable was number of lianas per tree (regressed on tree size and elevation) and when it was tree response as growth or survival (regressed on number of lianas per tree and tree size). This indicates that spatial aggregation of numbers of lianas per tree was low and near to random, that is, locally trees with many lianas and trees with few lianas were well mixed and not forming patches of similarly high or low liana-laden trees in the forest. This form of (spatial) aggregation is not to be confused with the (focal) aggregation of lianas themselves on individual trees (see Campbell and Newbery 1993). If tree growth and survival depended strongly on tree size, and trees in terms of their sizes were randomly distributed in the forest, local neighborhoods would not have shown correlated growth responses, again supporting a nearrandom distribution of responses spatially. The diffuse spread of lianas in and between crowns would also have diluted any loci of raised or lowered responses. The continual turnover of all size classes of focus trees and their neighbors, due to growth advancement, mortality, and recruitment, would moreover add further to the spatial variability as a form of neighborhood stochasticity (Newbery and Lingenfelder 2009, Newbery and Stoll 2013, Newbery and Ridsdale 2016). The lack of a spatial autocorrelation might suggest additionally that there were in fact very few large forest gaps created in recent decades, ones that had let in enough light to restart patchy secondary succession, and that the effects of thinning in the canopy during ENSO dry periods were broadly diffuse and scattered in terms of 
the temporarily enhanced light levels reaching the forest understory.

\section{Lianas in late succession}

Forest successional development was most likely different in MP1 and MP2 up until 1988. To have more lianas by then, on the very large trees in MP1 than in MP2, might indicate contingent historical processes, which implies that MP1 was perhaps more open than MP2 in the past. As a consequence, MP1 gained more lianas than MP2, and these were carried on up to 1988. If the thesis that the forest at Danum is now in an endsuccessional phase after a major disturbance $\sim 120 \mathrm{yr}$ prior to the first liana census is true (Newbery et al. 1992), and since then intermittently ENSO events have been occasionally thinning the overstory through small branch and twig abscission and thereby lighting the understory (Newbery et al. 1999, 2011, Walsh and Newbery 1999), liana densities and dynamics reflect an asynchrony between the plots in their successional recovery. Once lianas are well-established, ENSO events probably provide the means to put on more mass in the crowns of all stories. The two strong events in this study period were the 1982 1983 and 1997-1998 ENSOs (Newbery and Lingenfelder 2009), but moderate ones also occurred in 2010-2011 and 2015-2016 (based on an extension of the Accumulated Rainfall Anomaly index of Newbery and Lingenfelder 2009: Fig. 1). The first event came 3-4 yr before the main plots census in 1986 and was likely influential on the 1988 liana densities. Nunes et al. (2019) have shown that during the last event there was an increase in leaf litterfall at Danum.

This long-term forest process was probably continuing to converge between 1988 and 2018, with plot-level tree basal area moving toward its maximum faster in, or more ahead of, MP2 than in MP1. Mature closed forests approaching, or already at, the site-defined maximum carrying capacity (Newbery et al. 1992) might be expected to lessen their liana loads because as the forest became denser in basal area terms, branches that fell would be less easily replaced on the large trees in the overstory, and lianas would have increasingly less favorable light conditions in which to recruit and grow on the small and medium-sized trees. Heavy liana cover in the understory would further limit light reaching tree leaves and hasten their decline under competition with less infested neighbors (Schnitzer and Bongers 2011). By contrast, any large branch fall also perturbs the canopy by causing small openings, and these let in more light to the understory and hence promote tree and liana growth.

Species with high mean numbers of lianas per tree in 1988 had much larger decreases by 2018 than those with low numbers per tree (e.g., from 3.0 vs. 1.0 to $\sim 1.9$ vs. 0.9 ). This indicated a form of negative density dependence at the community level, perhaps by the more heavily laden species disproportionally shedding more lianas or dying from them, or recruiting them more slowly. However, species' numbers of lianas per tree in 1988, in 2018, and the differences between censuses, were not correlated with mortality (\%d) or growth rate of stems (rgr). Tree recruitment into the $\geq 30 \mathrm{~cm}$ gbh population might have been expected to have been highly correlated with growth rates of small trees (10 to $<50 \mathrm{~cm}$ gbh). This suggests that each species was individually determined by different combinations of the factors, which led to their gains and losses of lianas.

The increased separation of a group of several of the dipterocarp species in terms of numbers of lianas per tree and OUI, or similarly $\ln (\mathrm{gbh})$, between 1988 and 2018, highlighted an interesting dynamic conditional on a strong family trait. Differences in numbers between censuses reflected how the frequency distributions were changing with increasing size class. Even if branch shedding remains the leading hypothesis, other factors are important, for example, the tendency for medium-sized dipterocarps to have faster stem growth rates than most other species, which is contributory to their upward advancement toward the canopy (Newbery et al. 1999).

The species-level analysis revealed four species, which did not belong to the group with low numbers of lianas. Shorea fallax had not so low numbers but an intermediate OUI, while $S$. pauciflora had a high mean number of lianas despite its high OUI; $V$. dulitensis and $H$. nervosa were intermediate in both numbers of lianas and OUI values. Furthermore, S. pauciflora unlike the other dipterocarps barely changed between censuses. These differences may be explained as follows: S. fallax was unusual among the dipterocarps by having a large proportion of medium-sized trees 
and hence higher than average numbers of lianas per tree for a dipterocarp, and $V$. dulitensis and $H$. nervosa, though having a tendency to be medium-sized trees too, did not reach the maximum sizes of other dipterocarps (hence all three had intermediate OUIs; D. M. Newbery, P. Stoll, and M. Lingenfelder, unpublished data). Shorea pauciflora is somewhat special in that its maximum height was only $48.4 \mathrm{~m}$ vs. $51.0-62.2 \mathrm{~m}$ (mean $57.2 \mathrm{~m}$ ) for four others in the separated group (S. argentifolia, S. johorensis, S. parvifolia, and S. pilosa; D. M. Newbery, unpublished height data), and the corresponding height/dbh ratios were 0.36 vs. $0.45-0.51$ (mean 0.47 ). This height difference of $8.8 \mathrm{~m}$ may then account for the higher load of unshed lianas on S. pauciflora. Once again, the details of each species' dynamics, population structure, and architecture are all important. That the graphs of number of lianas per tree vs. $\ln$ (rgr) were similar to those for number of lianas vs. OUI with respect to the dipterocarps reflects the well-established association between fast growth of juveniles and final maximum size in the overstory.

Another reason why MP1 had more lianas on its very large trees than MP2 in 1988, might have been topography (see Fig. 1 in Lingenfelder and Newbery 2009). MP1 is on the side of a long pronounced ridge with steep slopes and is relatively topographically distinct, whereas MP2, also with an overall similar difference in elevation of 35$40 \mathrm{~m}$ over the $400 \mathrm{~m}$ plot length, is less steep and forms a spur to a higher backing ridge. These subtle differences in topography likely played a role in water availabilities during dry periods, the top of MP2 being less prone to dryness than the ridge top in MP1 (N. Chappell, personal communication). Past drought effects could ex hypothesis have been stronger in MP1 than in MP2, making larger lighter areas in the understory, and thus promoting the establishment of more lianas in the former than latter, with lasting consequences.

Although the number of lianas decreased with elevation, there was a similarly strong relationship in both plots: So differences in elevation-at least in recent decades - were not a good explanation for different liana changes across plots. Very large trees were also quite evenly spread across the topographic gradient in both plots. The proportions of the very largest trees $(\mathrm{gbh} \geq 240 \mathrm{~cm})$ dying in the lower and upper halves of the plots (corresponding roughly to lower slopes and ridges) were $35 \%$ and $32 \%$ in MP1 and $46 \%$ and $50 \%$ in MP2 $\left(\chi^{2}=0.05\right.$, $\left.P=0.82 ; \chi^{2}=0.09, P=0.77\right)$. So, host size distribution was not an explanation either. This would then indicate that MP2 probably recovered faster than MP1 in successional terms and any very large trees that did have high loads in MP2 had already fallen before 1988, and in the 1988-2018 period a similar phase was being realized in MP1. Meanwhile, MP2 was showing a next, maybe final, phase of liana loss within the understory. Indeed, if intensity of disturbance was less in MP2 than in MP1, a faster recovery (less impeded by lianas) would have been expected more in the former than the latter. This represents a shifting mosaic in time and space, in theory reminiscent of the pattern and process idea of Watt (1947), applied by Richards (1952) to rain forests. The dynamics of lianas in such a late-successional forest can be linked historically to liana activity soon after that distant strong disturbance set the longterm regrowth on its variable course.

\section{A new hypothesis for canopy roughness}

If during succession and forest maturity liana loads reach a quasi-stable equilibrium density and biomass (Muller-Landau and Visser 2019, MullerLandau and Pacala 2020), this would be expected in mid-succession when there remained sufficient light for recruitment and onward liana spread, and then followed decline as the forest canopy fully closed. By how much lianas might decline could depend on the time until a next major disturbance. If lianas established well on understory trees, they would respond positively to the ENSO dry periods and at the same time provide more cover for saplings and small trees of the canopy species during these perturbations, which reinforces the over-understory feedback model of Newbery et al. (1999). The ENSO pushes would offset the late-successional closing of the canopy (Newbery et al. 2011). As large trees matured and moved from the canopy into the emergent story, they may shed larger branches with heavy liana loads, or lianas are pulled away by being connected to neighboring trees that fall. This would free their crowns of light competition by lianas, and in dropping branches leave an increasing length of branch-free bole along which it would be difficult for lianas coming from lower crowns 
to cross onto and re-infest. Essentially, they could break free and escape. Large branch fall would, furthermore, drag connecting lianas to neighbors with them and thereby lessen the chance of reinfestation. The escape hypothesis provides an interesting explanation why this region of NE Borneo, relatively free of storms and below the cyclone belt, has so many standing deademergent trees (Gale and Hall 2001, Lingenfelder and Newbery 2009; D. M. Newbery, personal observation), and as a consequence why the canopies of forests of central Sabah are so obviously uneven (Newbery et al. 1992, 1999; D.M.N. reconnaissance flight). By contrast, smaller understory trees would not have been able to shed their lianas and continued to accumulate them over time until they died with the tree, a further burden for suppressed individuals. Being liana-free might also contribute to the stability of exceptionally tall dipterocarps recently recorded for Sabah (Jackson et al. 2021).

The liana shedding hypothesis would challenge Ashton and Hall's (1992) drought effects' one for canopy roughness of Bornean lowland dipterocarp forests, but it could also be seen to complement it if large trees lost large branches more frequently in dry periods than in wet ones. While lianas have the advantage of better growth in dry periods (Schnitzer 2005, 2018), for very large trees that response would eventually lead to their demise when their hosts shed them along with branches. A within-crown feedback process would downregulate liana abundances in a density-dependent manner. It is one mechanism that might operate in the host-liana (parasite) model of Muller-Landau and Pacala (2020). Since lianas do have strong competitive and growthsuppressing effects on trees, then species with the most successful survivors at the site can be expected to have evolved mechanisms to free themselves of their parasites. The cost of losing lower branches, measured in terms of temporarily reduced photosynthetic activity, should be less than the effect of sustaining a suppressing liana load that could limit the same longer term. Losing branches would also allow more light reaching juvenile trees in the understory, which, in the Dipterocarpaceae especially, tend to be clustered around their adults at Danum (Stoll and Newbery 2005). Pattern and extent of branch fall call for more detailed field recording and a clearer interpretation within a framework of tree architecture, branch growth, and survival (Rutishauser et al. 2011).

The liana shedding hypothesis places emphasis on how a host tree can minimize liana loads and improve its fitness, whereas the commoner line in the literature has been more about how the lianas can maximize their hold on hosts and their fitness. Ultimately, this would be played out in their respective life-history schedules. However, to properly model equilibrium dynamics, the inherently stochastic long- and short-term nature of the environment of N.E. Borneo-in the form of recoveries from past major disturbances and continuing ENSO perturbations, needs to be incorporated.

\section{ACKNOWLEDGMENTS}

We thank R. Ewers of Imperial College London for arrangements for the MSc work of C.Z. at Danum, R. Nilus (Sabah Forest Department) for local collaboration, Anthony F. Karolus for field assistance (Danum Valley Field Centre), and M. Lingenfelder (Bern University) for the Danum tree database management and taxonomic codes matching. Permission for C.Z. to conduct the fieldwork was granted by the Danum Valley Management Committee and the Sabah Biodiversity Council. Two anonymous reviewers are thanked for their improvements to the paper. D.M.N. designed the setup at Danum and conceived the study. C.Z. undertook the 2018 liana field census, the first analyses, and prepared the $\mathrm{R}$ code for the randomization procedure. D.M.N. extended the statistical analysis and interpretation, and largely wrote the paper. The first liana census in 1988, conducted by E. J. F. Campbell, was an extension to tree plot work made with a grant from the Natural Research Council in the UK (GR3/5555), and the analysis of the second liana census data drew on results of the main plot tree censuses to 2007 funded more recently by the Swiss National Science Foundation (Grants 3100-59088 and 31003A110250). The last plot census in 2015 for the trees was financially supported by the Chair for Vegetation Ecology (D.M.N.) at Bern.

\section{Literature Cited}

Agresti, A. 2007. An introduction to categorical data analysis. Second edition. J. Wiley \& Sons, Hoboken, New Jersey, USA.

Alam, M., L. Ronnegard, and X. Shen. 2015. Fitting conditional and simultaneous autoregressive spatial models in hglm. R Journal 7:5-18. 
Ashton, P. S. 1964. Ecological studies in the mixed dipterocarp forests of Brunei State. Oxford Forestry Memoirs 25, Oxford, UK.

Ashton, P. 2014. On the forests of tropical Asia. Kew Publishing, Royal Botanic Gardens, Kew, UK, and the Arnold Arboretum of Harvard University, Boston, Massachusetts, USA.

Ashton, P. S., and P. Hall. 1992. Comparisons of structure among mixed dipterocarp forests of northwestern Borneo. Journal of Ecology 80:459-481.

Augustin, N. H., M. A. Mugglestone, and S. T. Buckland. 1996. An autologistic model for the spatial distribution of wildlife. Journal of Applied Ecology 33:339-347.

Beaman, R. S., J. H. Beaman, C. W. Marsh, and P. V. Woods. 1985. Drought and forest fires in Sabah in 1983. Sabah Society Journal 8:10-30.

Besag, J. E. 1972. Nearest-neighbour systems and the auto-logistic model for binary data. Journal of the Royal Statistical Society Series B-Statistical Methodology 34:75-83.

Bivand, R. S., E. Pebesma, and V. Gomez-Rubio. 2013. Applied spatial data analysis with R. Springer, New York, New York, USA.

Bongers, F., C. E. N. Ewango, M. T. van der Sande, and L. Poorter. 2020. Liana species decline in Congo basin contrasts with global patterns. Ecology 101:1-10.

Borcard, D., F. Gillet, and P. Legendre. 2018. Numerical ecology with R. Springer, New York, New York, USA.

Brostrom, G., and H. Holmberg. 2011. Generalized linear models with clustered data: fixed and random effects models. Computational Statistics \& Data Analysis 55:3123-3134.

Caballe, G., and A. Martin. 2001. Thirteen years of change in trees and lianas in a Gabonese rainforest. Plant Ecology 152:167-173.

Cai, Z. Q., S. A. Schnitzer, and F. Bongers. 2009. Seasonal differences in leaf-level physiology give lianas a competitive advantage over trees in a tropical seasonal forest. Oecologia 161:25-33.

Cameron, A. C., and P. K. Trivedi. 2013. Regression analysis of count data. Second edition. Cambridge University Press, Cambridge, UK.

Campbell, E. J. F. 1990. The ecological relationship between lianas and trees in a lowland dipterocarp forest in Sabah, East Malaysia. Thesis. University of Stirling, Stirling, UK.

Campbell, E. J. F., and D. M. Newbery. 1993. Ecological relationships between lianas and trees in lowland rain forest in Sabah, East Malaysia. Journal of Tropical Ecology 9:469-490.

Clark, D. B., and D. A. Clark. 1990. Distribution and effect on tree growth of lianas and woody hemiepiphytes in a Costa Rican tropical wet forest. Journal of Tropical Ecology 6:321-331.

Cliff, A. D., and J. K. Ord. 1981. Spatial processes: models and applications. Pion, London, UK.

Cohen, J. 1988. Statistical power analysis for the behavioral sciences. Lawrence Erlbaum, Mahwah, New Jersey, USA.

Cohen, J. 1992. A power primer. Psychological Bulletin 112:155-159.

Cox, C. J., W. Edwards, M. J. Campbell, W. F. Laurance, and S. G. W. Laurance. 2019. Liana cover in the canopies of rainforest trees is not predicted by local ground-based measures. Austral Ecology 44:759-767.

DeWalt, S. J., et al. 2015. Biogeographical patterns of liana abundance and diversity. Pages 131-146 in S. A. Schnitzer, F. Bongers, R. J. Burnham, and F. E. Putz, editors. Ecology of lianas. J. Wiley \& Sons, Chichester, UK.

DeWalt, S. J., S. A. Schnitzer, and J. S. Denslow. 2000. Density and diversity of lianas along a chronosequence in a central Panamanian lowland forest. Journal of Tropical Ecology 16:1-19.

Dormann, C. F. 2007. Assessing the validity of autologistic regression. Ecological Modelling 207:234242.

Evans, J. S. 2018. spatialEco. R package (version 1.3-1). https://github.com/jeffreyevans/spatialEco

Fox, J., and S. Weisberg. 2011. An R companion to applied regression. Second edition. Sage, Los Angeles, California, USA.

Gale, N., and P. Hall. 2001. Factors determining the modes of tree death in three Bornean rain forests. Journal of Vegetation Science 12:337-346.

Gentry, A. H. 1991. The distribution and evolution of climbing plants. Pages 3-42 in F. E. Putz and H. A. Mooney, editors. The biology of vines. CUP, Cambridge, UK.

Haining, R. 2010. Spatial data analysis: theory and practice. CUP, Cambridge, UK.

Hazebroek, H. P., T. Z. Adlin, and W. Sinun. 2012. Danum Valley: the rain forest. Natural History Publications (Borneo), Kota Kinabalu, Sabah, Malaysia.

Hegarty, E. E. 1991. Vine-host interactions. Pages 357375 in F. E. Putz and H. A. Mooney, editors. The biology of vines. CUP, Cambridge, UK.

Hilbe, J. M. 2009. Logistic regression models. CRC Press/Chapman and Hall, Boca Raton, Florida, USA.

Hilbe, J. M. 2011. Negative binomial regression. Cambridge University Press, Cambridge, UK.

Ingwell, L. L., S. J. Wright, K. K. Becklund, S. P. Hubbell, and S. A. Schnitzer. 2010. The impact of lianas on 10 years of tree growth and mortality on Barro 
Colorado Island, Panama. Journal of Ecology 98:879-887.

Jackson, T. D., et al. 2021. The mechanical stability of the world's tallest broadleaf trees. Biotropica 53:110-120.

Lee, Y., J. A. Nelder, and Y. Pawitan. 2017a. Generalized linear models with random effects: unified analysis via H-likelihood. Second edition. CRC Press, Boca Raton, Florida, USA.

Lee, Y., L. Rönnegard, and M. Noh. 2017b. Data analysis using hierarchical generalized linear models with R. CRC Press, Boca Raton, Florida, USA.

Lingenfelder, M., and D. M. Newbery. 2009. On the detection of dynamic responses in a droughtperturbed tropical rainforest in Borneo. Plant Ecology 201:267-290.

Long, J. S., and J. Freese. 2014. Regression models for categorical dependent variables using Stata. Third edition. Stata Press, College Station, Texas, USA.

Malhi, Y., and O. L. Phillips, editors. 2005. Tropical forests and global atmospheric change. Oxford University Press, Oxford, UK.

Manly, B. F. J. 1997. Randomization, bootstrap and Monte Carlo methods in biology. Second edition. Chapman and Hall, London, UK.

Marsh, C. W., and A. G. Greer. 1992. Forest land-use in Sabah, Malaysia: and introduction to the Danum Valley. Philosophical Transactions of the Royal Society of London Series B-Biological Sciences 335:331-339.

Muller-Landau, H., and S. W. Pacala. 2020. What determines the abundance of lianas and vines? in A. P. Dobson, R. D. Holt, and D. Tilman, editors. Unsolved problems in ecology. Princeton University Press, Princeton, New Jersey, USA.

Muller-Landau, H. C., and M. D. Visser. 2019. How do lianas and vines influence competitive differences and niche differences among tree species? Concepts and a case study in a tropical forest. Journal of Ecology 107:1469-1481.

Newbery, D. M., E. J. F. Campbell, Y. F. Lee, C. E. Ridsdale, and M. J. Still. 1992. Primary lowland dipterocarp forest at Danum Valley, Sabah, Malaysia: structure, relative abundance and family composition. Philosophical Transactions of the Royal Society of London Series B-Biological Sciences 335:341-356.

Newbery, D. M., E. J. F. Campbell, J. Proctor, and M. J. Still. 1996. Primary lowland dipterocarp forest at Danum Valley, Sabah, Malaysia. Species composition and patterns in the understorey. Vegetatio 122:193-220.

Newbery, D. M., D. N. Kennedy, G. H. Petol, L. Madani, and C. E. Ridsdale. 1999. Primary forest dynamics in lowland dipterocarp forest at Danum Valley, Sabah, Malaysia, and the role of the understorey. Philosophical Transactions of the Royal Society B-Biological Sciences 354:1763-1782.

Newbery, D. M., and M. Lingenfelder. 2009. Plurality of tree species responses to drought perturbation in Bornean tropical rain forest. Plant Ecology 201:147-167.

Newbery, D. M., M. Lingenfelder, K. F. Poltz, R. C. Ong, and C. E. Ridsdale. 2011. Growth responses of understorey trees to drought perturbation in tropical rainforest in Borneo. Forest Ecology and Management 262:2095-2107.

Newbery, D. M., and C. E. Ridsdale. 2016. Neighbourhood abundance and small-tree survival in a lowland Bornean rainforest. Ecological Research 31:353-366.

Newbery, D. M., and P. Stoll. 2013. Relaxation of species-specific neighborhood effects in Bornean rain forest under climatic perturbation. Ecology 94:2838-2851.

Nunes, M. H., et al. 2019. Changes in leaf functional traits of rainforest canopy trees associated with an El Nino event in Borneo. Environmental Research Letters 14:1-14.

Phillips, O. L., et al. 2002. Increasing dominance of large lianas in Amazonian forests. Nature 418:770774.

Phillips, O. L., R. V. Martinez, A. M. Mendoza, T. R. Baker, and P. N. Vargas. 2005. Large lianas as hyperdynamic elements of the tropical forest canopy. Ecology 86:1250-1258.

Pinard, M. A., and F. E. Putz. 1994. Vine infestation of remnant trees in logged forest in Sabah, Malaysia: biomechanical facilitation in vine succession. Journal of Tropical Forest Science 6:302-309.

Pinheiro, J. C., and D. M. Bates. 2000. Mixed-effects models in S and S-plus. Springer, New York, New York, USA.

Plant, R. E. 2012. Spatial data analysis in ecology and agriculture using R. CRC Press, Boca Raton, Florida, USA.

Putz, F. E. 1984. How trees avoid and shed lianas. Biotropica 16:19-23.

Putz, F. E., and P. Chai. 1987. Ecological studies of lianas in Lambir National Park, Sarawak, Malaysia. Journal of Ecology 75:523-531.

R Core Team. 2020. R: a language and environment for statistical computing. R Foundation for Statistical Computing, Vienna, Austria.

Rhodes, J. R., C. A. McAlpine, A. F. Zuur, G. M. Smith, and E. N. Ieno. 2009. GLMM applied on the spatial distribution of koalas in a fragmented landscape. Pages 469-492 in A. F. Zuur, E. N. Ieno, N. J. Walker, A. A. Saveliev, and G. M. Smith, editors. Mixed effects models and extensions in ecology with R. Springer, New York, New York, USA. 
Richards, P. W. 1952. The tropical rain forest. First edition. Cambridge University Press, Cambridge, UK.

Richards, P. W. 1996. The tropical rain forest. Second edition. Cambridge University Press, Cambridge, UK.

Ronnegard, L., X. Shen, and M. Alam. 2010. Hglm: a package for fitting hierarchical generalized linear models. R Journal 2:20-28.

Rutishauser, E., D. Barthelemy, L. Blanc, and E. A. Nicolini. 2011. Crown fragmentation assessment in tropical trees: method, insights and perspectives. Forest Ecology and Management 261:400-407.

Schnitzer, S. A. 2005. A mechanistic explanation for global patterns of liana abundance and distribution. American Naturalist 166:262-276.

Schnitzer, S. A. 2018. Testing ecological theory with lianas. New Phytologist 220:366-380.

Schnitzer, S. A., and F. Bongers. 2002. The ecology of lianas and their role in forests. Trends in Ecology \& Evolution 17:223-230.

Schnitzer, S. A., and F. Bongers. 2011. Increasing liana abundance and biomass in tropical forests: emerging patterns and putative mechanisms. Ecology Letters 14:397-406.

Schnitzer, S. A., and W. P. Carson. 2010. Lianas suppress tree regeneration and diversity in treefall gaps. Ecology Letters 13:849-857.

Sheil, D., and R. M. May. 1996. Mortality and recruitment rate evaluations in heterogeneous tropical forests. Journal of Ecology 84:91-100.

Stoll, P., and D. M. Newbery. 2005. Evidence of speciesspecific neighborhood effects in the Dipterocarpaceae of a Bornean rain forest. Ecology 86:3048-3062.

Swaine, M. D., and J. Grace. 2007. Lianas may be favoured by low rainfall: evidence from Ghana. Plant Ecology 192:271-276.

Thomas, D., R. J. Burnham, G. Chuyong, D. Kenfack, and M. N. Sainge. 2015. Liana abundance and diversity in Cameroon's Korup National Park. Pages 13-22 in S. A. Schnitzer, F. Bongers, R. J. Burnham, and F. E. Putz, editors. Ecology of lianas. J. Wiley \& Sons, Chichester, UK.

van der Heijden, G. M. F., J. R. Healey, and O. L. Phillips. 2008. Infestation of trees by lianas in a tropical forest in Amazonian Peru. Journal of Vegetation Science 19:747-756.

van der Heijden, G. M. F., and O. L. Phillips. 2009. Liana infestation impacts tree growth in a lowland tropical moist forest. Biogeosciences 6:2217-2226.

ver Hoef, J. M., and P. L. Boveng. 2007. Quasi-poisson vs. negative binomial regression: How should we model overdispersed count data? Ecology 88:27662772.

Visser, M. D., H. C. Muller-Landau, S. A. Schnitzer, H. de Kroon, E. Jongejans, and S. J. Wright. 2018a. A host-parasite model explains variation in liana infestation among co-occurring tree species. Journal of Ecology 106:2435-2445.

Visser, M. D., S. A. Schnitzer, H. C. Muller-Landau, E. Jongejans, H. de Kroon, L. S. Comita, S. P. Hubbell, and S. J. Wright. 2018b. Tree species vary widely in their tolerance for liana infestation: a case study of differential host response to generalist parasites. Journal of Ecology 106: 781-794.

Walsh, R. P. D. 1996a. Climate. Pages 159-236 in P. W. Richards [1996] The tropical rain forest: an ecological study. Cambridge University Press, Cambridge, UK.

Walsh, R. P. D. 1996b. Drought frequency changes in Sabah and adjacent parts of northern Borneo since the late nineteenth century and possible implications for tropical rain forest dynamics. Journal of Tropical Ecology 12:385-407.

Walsh, R. P. D., and D. M. Newbery. 1999. The ecoclimatology of Danum, Sabah, in the context of the world's rainforest regions, with particular reference to dry periods and their impact. Philosophical Transactions of the Royal Society of London Series B-Biological Sciences 354:1869-1883.

Watt, A. S. 1947. Pattern and process in the plant community. Journal of Ecology 35:1-22.

Whitmore, T. C. 1984. Tropical rain forests of the Far East. Oxford Science Publications, Oxford, UK.

Wright, S. J. 2005a. The El Nino Southern Oscillation influences tree performance in tropical rainforests. Pages 295-310 in E. Birmingham, C. W. Dick, and C. Moritz, editors. Tropical rainforests: past, present, and future. Chicago University Press, Chicago, Illinois, USA.

Wright, S. J. 2005b. Tropical forests in a changing environment. Trends in Ecology \& Evolution 20:553560.

Wright, S. J., O. Calderon, A. Hernandez, and S. Paton. 2004. Are lianas increasing in importance in tropical forests? A 17- year record from Panama. Ecology 85:484-489.

Wright, S. J., I. F. Sun, M. Pickering, C. D. Fletcher, and Y. Y. Chen. 2015. Long-term changes in liana loads and tree dynamics in a Malaysian forest. Ecology 96:2748-2757.

Zahnd, C. 2018. Long-term change in liana infestation in a Bornean rainforest. Thesis. Imperial College London, London, UK.

Zuur, A. F., E. N. Ieno, N. J. Walker, A. A. Saveliev, and G. M. Smith. 2009. Violation of independence Part II. Pages 161-191 in A. F. Zuur, E. N. Ieno, N. J. Walker, A. A. Saveliev, and G. M. Smith, editors. Mixed effects models and extensions in ecology with R. Springer, New York, USA. 


\section{Data Avallability}

The data from the two liana censuses are available at the Dryad Digital Repository (https://doi.org/10.5061/ dryad.z8w9ghx8t).

\section{SUPPORTING INFORMATION}

Additional Supporting Information may be found online at: http://onlinelibrary.wiley.com/doi/10.1002/ecs2. 3537/full 